\title{
Alternating-gradient canted cosine theta superconducting magnets for future compact proton gantries
}

\author{
Weishi Wan, ${ }^{1, *}$ Lucas Brouwer, ${ }^{1,2}$ Shlomo Caspi, ${ }^{1}$ Soren Prestemon, ${ }^{1}$ Alexander Gerbershagen, ${ }^{3}$ \\ Jacobus Maarten Schippers, ${ }^{3}$ and David Robin ${ }^{1}$ \\ ${ }^{1}$ Lawrence Berkeley National Laboratory, 1 Cyclotron Road, Berkeley, California 94720, USA \\ ${ }^{2}$ Department of Nuclear Engineering, University of California, Berkeley, California 94720, USA \\ ${ }^{3}$ Paul Scherrer Institut, 5232 Villigen PSI, Switzerland \\ (Received 13 February 2015; published 23 October 2015)
}

\begin{abstract}
We present a design of superconducting magnets, optimized for application in a gantry for proton therapy. We have introduced a new magnet design concept, called an alternating-gradient canted cosine theta (AG-CCT) concept, which is compatible with an achromatic layout. This layout allows a large momentum acceptance. The $15 \mathrm{~cm}$ radius of the bore aperture enables the application of pencil beam scanning in front of the SC-magnet. The optical and dynamic performance of a gantry based on these magnets has been analyzed using the fields derived (via Biot-Savart law) from the actual windings of the AG-CCT combined with the full equations of motion. The results show that with appropriate higher order correction, a large 3D volume can be rapidly scanned with little beam shape distortion. A very big advantage is that all this can be done while keeping the AG-CCT fields fixed. This reduces the need for fast field ramping of the superconducting magnets between the successive beam energies used for the scanning in depth and it is important for medical application since this reduces the technical risk (e.g., a quench) associated with fast field changes in superconducting magnets. For proton gantries the corresponding superconducting magnet system holds promise of dramatic reduction in weight. For heavier ion gantries there may furthermore be a significant reduction in size.
\end{abstract}

\section{INTRODUCTION}

In an accelerator-based ion-beam particle therapy (IBT) facility, ions (typically protons and/or carbon ions) are accelerated and injected into patients' bodies to treat deepseated cancer tumors [1]. Many IBT facilities use rotatable gantry beam lines to direct the ion beam at the patient from different angles. The ability of gantries to direct the beam into the body from different angles allows for using the combination of angles that will minimize the radiation dose to healthy tissue. Different angles of entrance are also used to avoid hitting with the beam sensitive organs, such as spine, blood vessels and heart.

The trend in modern proton medical therapy is to employ gantry systems with the so-called pencil beam scanning technique. This technique potentially gives the best possible dose distribution. In the scanning approach, the energy deposition is highly focused and controlled, allowing for exquisite accuracy in treatment and minimal collateral damage to healthy tissue. The depth of scanning can be adjusted by changing the beam energy, whereas the beam

\footnotetext{
*wwan@1bl.gov

Published by the American Physical Society under the terms of the Creative Commons Attribution 3.0 License. Further distribution of this work must maintain attribution to the author $(s)$ and the published article's title, journal citation, and DOI.
}

transverse position can be rapidly changed using fast sweeper magnets located upstream and/or downstream of the final bending magnets. In gantries [2,3] developed at PSI during the past 20 years, the scanning technique has been developed and significant experience has been obtained in the routine clinical application of this technique.

The advantages of gantries are such that most of the centers use them in many if not all of their treatment rooms. The current state of the art for pencil beam scanning gantries are Gantry 2 at PSI [4] [proton; see Fig. 1 (top)] and the gantry at the Heidelberg Ion-Beam Therapy Center (HIT) $[5,6]$ (carbon). The performance of these gantries is highly advanced (see Sec. II).

As discussed in more detail in Ref. [7], there is significant interest in research to reduce the cost of the proton therapy facilities in order to make this therapy more affordable for patients, hospitals and health insurance companies. For the commercial application of proton and ion beam therapy, it has become evident that the weight, size (and thus price) and scanning speed of current scanning gantries impose major obstacles for the introduction of the scanning technique in a hospital-based environment.

In order to reduce the size of a gantry, the magnetic field of the bending magnet has to be increased. For the field higher than $1.8 \mathrm{~T}$, the saturation of the iron yoke causes the weight of a resistive bending magnet to be prohibitively large. As a result, superconducting magnet technology 

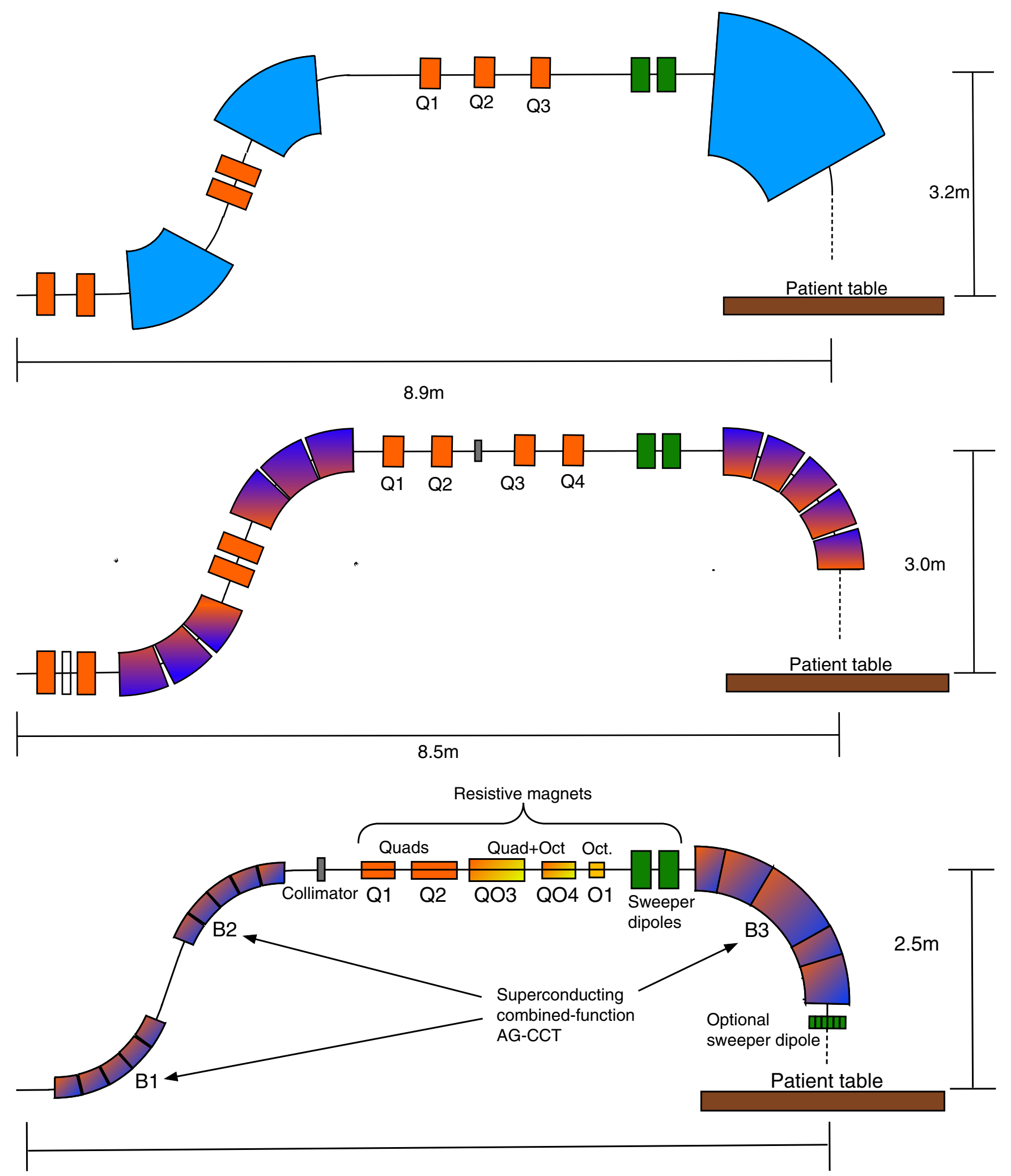

$8.3 m$

FIG. 1. Three gantry layouts: PSI gantry II (top); a possible achromatic design, as presented in [7] (middle); the layout developed in this paper, i.e., an achromatic gantry using three AG-CCT magnets (bottom). The magnets shaded with two colors are of a combined function type: dipole + quadrupole fields. 
becomes the only potentially viable choice. The fact that superconducting magnets can double or triple the bending field entails that the size of the gantry can be significantly reduced. The more striking consequence of employing superconducting magnet technology is the dramatic reduction of the weight of the bending magnets, since most of the field is generated by the superconducting coils and hence much less iron is needed.

Apart from increased complexity in technology, the main drawback of the superconducting magnets is their slow ramping speed of the field compared to that of the resistive magnets. To that end, our previous design [8] using weak focusing superconducting bending magnets and the superconducting carbon gantry being developed at NIRS/Toshiba [9-12] are both limited in depth scanning speed.

One way to increase depth scanning speed is to design a gantry with larger momentum acceptance so that the magnetic field can remain fixed when energy of the beam is changed. Towards that end, two types of designs have emerged over the past few years. The first type can be considered a variant of the state of the art gantries [4-6], where the homogeneous bending magnets are replaced with achromatic bending sections [7,13-15]. As a result, dispersion is significantly reduced and momentum acceptance is increased. Among them, the design that is being realized at ProNova $[13,14]$ can increase momentum acceptance to a few percent, roughly 5 to 10 times larger than the typical $(<1 \%)$ momentum acceptance of normalconducting gantries. It allows the gantry to ramp the field at a much reduced speed at any given gantry position.

The second type represents a more radical departure from the existing gantries [16,17]. Employing the nonscaling Fixed-Field Alternating-Gradient (FFAG) concept, this new type of gantries has momentum acceptance up to $40 \%-50 \%$, potentially eliminating the need of ramping the field all together for most cases. Due to the strong focusing, the beam width remains small for all energies, allowing magnets to have a very small bore and hence very light weight. The main challenge for this type of gantry is the magnet design showing the feasibility of building these magnets.

In this paper we introduce a new superconducting magnet concept that we call a left-right canted cosine theta (LR-CCT) magnet. When applied to a quadrupole CCT winding, this approach yields an alternating-gradient (i.e., quadrupole) field (AG-CCT). Such a magnet can be combined with a dipole CCT to provide compact, achromatic, large momentum acceptance $(\sim 25 \%)$ bending sections. We present the design of a compact gantry using curved superconducting AG-CCT magnets. We then show that with such a gantry, it is possible, without changing the fields of the AG-CCT magnets, to enable pencil beam scanning of a proton beam over a large volume (both transverse and depth) with minimal distortion of the beam shape. This allows for rapid 3D scanning. Furthermore we estimate that the weight of the final AG-CCT magnet will be reduced by an order of magnitude compared to normal conducting magnets, e.g., such as the one used at PSI [4]. In a word, our new design results in a gantry with (i) large (an order of magnitude) weight reduction, (ii) possible size reduction (particularly for ion gantries), and (iii) rapid 3D scanning.

From the point of view of either layout or beam optics, our design is much closer to the first type mentioned above, with a larger momentum acceptance, which is a direct result of our novel magnet design. The room between the localized achromatic bending sections allows one to place beam collimators to better define the beam as well as scanners to scan the beam. Our design can definitely be characterized as a locally achromatic FFAG type, since the field of the superconducting magnets remains unchanged at any given field for most cases.

In Sec. II, we start with an overview of desired gantry properties, followed by a short discussion on the present state of the art and limitations of scanning gantries. Next, the concept of a curved achromatic section consists of five combined-function fixed-field alternating-gradient (FDFDF) magnets is presented and the way such an FDFDF section can be incorporated into a gantry to provide a large momentum acceptance is discussed. In Sec. III, we introduce the general concept of the AG-CCT magnet and show the reasons that such a magnet is an efficient and effective way to realize such an FDFDF section. In Sec. IV, we discuss how a compact gantry can be be designed using three AG-CCT magnet sections. We then present the linear optical properties of the design that satisfy the requirements listed in Sec. II. In Sec. V, we provide details of the magnetic design of the three AG-CCT sections including real field profiles. In Sec. VI, we present the beam dynamic simulations of the gantry including the full magnetic fields of the magnets and all kinematic effects. In this section we demonstrate the feasibility of such a gantry design to scan a large volume while maintaining minimal beam distortion while keeping the fields of the AG-CCTs fixed.

\section{DESIRED GANTRY PROPERTIES AND ACHROMATIC GANTRY DESIGNS}

The purpose of the proton therapy gantry is to rotate the beam around the patient to irradiate the tumor from different angles. Modern proton therapy gantries typically scan the beam within the tumor volume in all three dimensions by modifying the transverse position and energy of the beam. In this section we will summarize some of the main desired properties of gantries. Next we will describe the state of the art of existing gantries. This is followed by a discussion of the definition and importance of an achromat and the impact on the size of the momentum acceptance. Finally we introduce the concept of a large momentum acceptance gantry built of achromatic FFAG sections. 


\section{A. Desired functional properties}

The requirements of the new gantries have been discussed in detail in [7]. Here we list some of the desired beam, gantry, and scanning properties that impact the design and performance of gantries.

\section{Proton beam size}

The typical $2 \sigma$ acceptance of the proton beam is $10-30 \pi \mathrm{mm}$-mrad in both transverse planes. For instance for PSI, Gantry 2, a $30 \pi$ mm-mrad $2 \sigma$ acceptance has been chosen. A large acceptance is needed to comply with the large emittance of the proton beams that have been degraded to the appropriate energy in the preceding beam transport system. A large acceptance of the beam transport system and the gantry is essential to keep sufficient beam intensity at the patient.

As in most gantries, we have assumed a dispersion free beam with equal beam emittances in the two transversal planes at the coupling point of the gantry to the beam line. This assures that the beam in the gantry is independent of the gantry's rotation angle. The beam spot at the isocenter should be dispersion free, round and Gaussian-like shaped with a $(2 \sigma)$ radius of approximately $4-6 \mathrm{~mm}$.

\section{Gantry space and motion constraints}

The gantry must be able to rotate by at least an angle of $180^{\circ}$, but typically by $360^{\circ}$, around the patient with a distance of the $\approx 50 \mathrm{~cm}$ from the beam line exit (gantry nozzle) to the isocenter. An additional $\approx 50 \mathrm{~cm}$ space downstream of the final bend is occupied by the beam diagnostics and dosimetry equipment. Consequently, at least one meter distance must be reserved between the end of the final bending magnet and the isocenter.

\section{Scanning}

The transverse scanning should cover a large transverse area (called "scanning field") at the isocenter plane. The scanning field should be greater than $20 \mathrm{~cm} \times 20 \mathrm{~cm}$ and ideally $30 \mathrm{~cm} \times 40 \mathrm{~cm}$. The scanning can be performed with fast normal conducting sweeper magnets to shift the pencil beam with a speed of a few $\mathrm{cm} / \mathrm{ms}$ in the isocenter using reasonable magnetic fields (maximum deflection of up to $80 \mathrm{mrad}$ ). The round beam spot formed at the isocenter should not be distorted by the scanning.

The scanning magnets can be located upstream or downstream of the final bending magnet. The downstream scanning has an advantage that the final bending magnet can have a relatively small aperture. For upstream scanning, this magnet's aperture must be large, in order to allow the scanned beam trajectories to pass. However, in case of upstream scanning the total radius of the gantry is typically lower since the beam optics can be designed to have an approximate point-to-parallel imaging from the sweeper magnets to the isocenter.
An advantage of the upstream scanning is the lower skin dose that is obtained with parallel pencil beam displacements. In downstream scanning, one needs $1.5-2 \mathrm{~m}$ distance (so-called SAD: source-to-axis-distance) between the sweeper magnets and the patient to achieve this, resulting in a larger gantry radius.

For the longitudinal or depth scanning by energy variation, the requirement is to modulate the depth of the Bragg peak in water between 4 and $30 \mathrm{~cm}$, corresponding to the proton beam energies of 70 to $220 \mathrm{MeV}$. Scanning is typically performed in layers of $5 \mathrm{~mm}$ depth $(5 \mathrm{~mm}$ depth corresponds to $1 \%$ change in beam momentum). To achieve a short enough treatment time, the corresponding energy step must be made rather fast. At PSI this is done within $100 \mathrm{~ms}$, currently the fastest system in the world. Hence, the gantry must be able to support the beam momentum change rate of $d p / d t=10 \% / \mathrm{s}$. In Table I we summarize the functional requirements.

\section{B. State of the art and limitations}

As previously mentioned, the current state of the art for pencil beam scanning gantries are the PSI Gantry 2 (proton; see Fig. 1) and the HIT gantry (carbon). Both of these gantries provide an impressive list of capabilities: (i) $180^{\circ}-$ $360^{\circ}$ achieved beam directions; (ii) small spot size at the isocenter; (iii) large source-to-axis distances (SAD) for nearly parallel scanning; (iv) fast transverse scanning speeds; (v) large $(>10 \mathrm{~cm} \times 10 \mathrm{~cm})$ good field regions; and (vi) 3-30 $\mathrm{cm}$ penetration depth (range).

In PSI Gantry 2, as well as in most normal conducting proton therapy gantries, the maximal value of the dispersion function is very high at one or more locations. Hence, at these locations the momentum acceptance is limited by the aperture of the gantry magnets and the vacuum pipe. The beams sent to the normal conducting

TABLE I. Summary of functional requirements.

\begin{tabular}{|c|c|c|}
\hline Parameter & Acceptable & Desirable \\
\hline Angular range of gantry $\left({ }^{\circ}\right)$ & 180 & 360 \\
\hline Proton acceptance & & \\
\hline$(2 \sigma)(\pi \mathrm{mm}-\mathrm{mrad})$ & 10 & 30 \\
\hline Beam size at isocenter $(2 \sigma)(\mathrm{mm})$ & 6 & 4 \\
\hline Beam energy range $(\mathrm{MeV})$ & 70 to 220 & 60 to 250 \\
\hline $\begin{array}{l}\text { Minimum space from the last } \\
\text { magnet to the isocenter }(\mathrm{m})\end{array}$ & 1 & 1 \\
\hline Minimum SAD (m) & 2 & 3 \\
\hline Maximum sweeper angle (mrad) & \pm 80 & \pm 60 \\
\hline Transverse scanning field $\left(\mathrm{cm}^{2}\right)$ & $20 \times 20$ & $30 \times 40$ \\
\hline Transverse scanning speed & few $\mathrm{cm} / \mathrm{ms}$ & few $\mathrm{cm} / \mathrm{ms}$ \\
\hline $\begin{array}{l}\text { Momentum range w/o ramping } \\
\text { superconducting magnets }(\%)\end{array}$ & $> \pm 10 \%$ & $\pm 50 \%$ \\
\hline Momentum scanning rate, $d p / d t$ & $10 \% / \mathrm{s}$ & $10 \% / \mathrm{s}$ \\
\hline $\begin{array}{l}\text { Transverse field scanning rate } \\
\text { (using } 5 \mathrm{~mm} \text { steps) }\end{array}$ & $1 \mathrm{step} / \mathrm{ms}$ & $1 \mathrm{step} / \mathrm{ms}$ \\
\hline
\end{tabular}


gantries are prepared such that their momentum spread is confined to $< \pm 0.5$ to $\pm 1 \%$. During the energy variation to distribute the dose in depth, the fields of all dipole and quadrupole magnets of the gantry must be set precisely proportionally to these beam momenta.

When considering the needed speed of energy changes, it is important to realize that the ramping speed of the magnets limits the maximal energy modulation rate. This would be a serious drawback for superconducting magnets, if no other measures are taken.

\section{Definition and importance of global and local achromatic gantry design}

Each dipole magnet of the beam line introduces dispersion of the beam. A beam with nonzero dispersion is required at the following two positions in a gantry: (i) at the coupling point, where the beam is transferred from the fixed beam line to the rotating gantry and (ii) at the isocenter, where target tissue is located. Nonzero dispersion at these positions would yield a correlation between the particles transverse position in the pencil-beam spot and their penetration depth in the tissue, making the irradiated spots asymmetric in $x-y$ plane. In addition the spot size may be too large.

An achromatic beam transport section is one where particles entering with different momentum but the same transverse position and angle will leave the section ending with the same position and angle. In other words there is no dispersion (or derivatives of dispersion) at the entrance and the exit of the section: most existing gantries are globally achromatic-from the coupling point to the isocenter. Application of superconducting technology makes it possible $[7,15]$ to arrive at compact locally achromatic curved sections, by which it is relatively easy to obtain a very large momentum acceptance. If these curved sections can be provided with large aperture (in particular before the isocenter) and acceptable optical properties, it would bring the functional requirements laid out in Sec. II A within reach.

\section{Combined-function alternating-gradient magnet sections}

Towards this end a new gantry design was introduced [7] where the achromatic curved section consists of five combined-function (FDFDF), fixed-field, large aperture, alternating-gradient magnets. This concept can be seen in the middle gantry in Fig. 1. It can be compared with the PSI Gantry 2 on the top. The original Gantry 2 optics has been changed from a global gantry design into a design with three local achromatic bending sections. Each bending section, however, consists of multiple quadrupoles and dipoles, some of which with a combined function (strong field gradient). The linear optics of this gantry is described in more detail in [7]. In that paper it is shown that the desired properties listed in Sec. II A are satisfied to first order. In this paper we address two outstanding issues: (i) a realizable single magnetic layout for the locally achromatic sections and (ii) a gantry design that satisfies the requirements listed in Sec. II A not just to first order but also considering all high order beam dynamic effects.

To achieve these goals a new gantry was designed (see the bottom of Fig. 1). This gantry is a variation on the design of the middle gantry shown in Fig. 1.

\section{Magnetic layout for the achromatic section}

One can imagine a compact and effective method of realizing such an FDFDF achromatic curved section would be to combine the full section into a single element rather than having five separate magnets. In such a scheme, the dipole field is distributed over the full section with the different focusing FDFDF sections butted up against each other and also extended over the full section. By doing this, the drifts are eliminated and the dispersion can be minimized. A gantry using a simple drawing of this concept can be seen in the bottom of Fig. 1. This gantry has locally achromatic (FDFDF) sections labeled B1, B2, and B3. The details of these sections as well as the beam dynamics of the full gantry will be the subject of this paper.

The question addressed is how to effectively realize such locally achromatic curved sections. It turns out that a natural way to do this is by extending the canted cosine theta (CCT) concept. We call this the alternating-gradientcanted cosine theta or AG-CCT. The AG-CCT concept and parameters for this gantry are discussed in Secs. III and V.

In Sec. IV we discuss the approach to the gantry design by presenting the simulation results including the full fields of the magnets as well as comprehensive beam dynamics results.

\section{A COMBINED-FUNCTION, EFFICIENT ALTERNATING-GRADIENT (AG-CCT) MAGNET}

Previous superconducting gantry design at LBNL has focused on the need for a curved combined function final bending magnet $[8,18]$. These studies have shown the feasibility and advantages of using the canted cosine theta (CCT) design [19-25] to generate desired field multipoles in a large aperture superconducting gantry magnet. Using the CCT approach, a novel magnet concept has been developed to produce the alternating focusing and defocusing fields needed for the achromatic gantry, which is described in detail in Sec. IV.

The new concept consists of multiple CCT quadrupole winding sections placed in sequence on a curve such that the effective current direction is reversed between sections. This produces alternating quadrupole field regions along the length of the bend whose individual integral strengths can be tuned by the location of the current polarity transitions. A simple transition scheme to reverse the current between sections has been developed to allow 

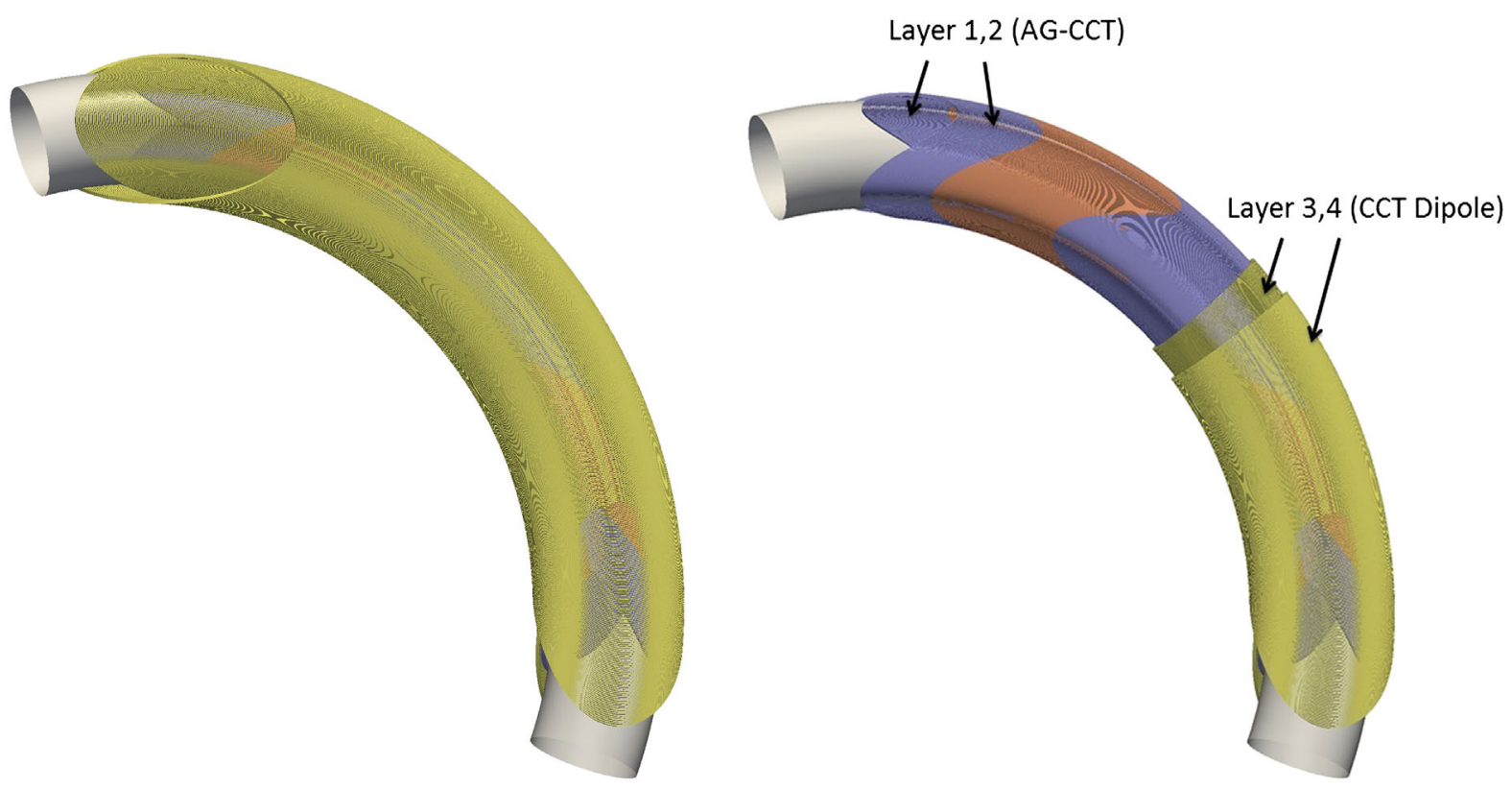

FIG. 2. Coil layers of curved $90^{\circ}$ AG-CCT (FDFDF) magnet. Left: The four layers of coils all overlayed. Right: Only about half of two outer dipole layers (Layer 3 and 4) are shown in order to see the two inner alternating quadrupole layers (Layer 1 and 2).

for the use of one continuous winding and power supply. In this scheme the ends of each section nest inside the next, resulting in an alternating focusing system that is both compact and efficient (see Fig. 2).

The method for changing the current between sections makes use of the axial periodicity of CCT windings, i.e., left or right-handed "corkscrew" winding pattern. Changing the direction of this period, e.g., switching from left- to right-handed corkscrew, switches the direction of the axial current and thus the transverse fields. After a winding path is optimized to produce a quadrupole (a method for field description and optimization will be discussed in Sec. VI), the coil can be split up into sections of alternating axial period based on the desired integrated strengths of the focusing and defocusing regions. Once the coil sections are determined, only a short reverse bend is needed to transition between regions and maintain one continuous winding. The result is a highly efficient transition from focusing to defocusing quadrupole.

We note that the same approach can be used for dipole windings to produce fields of opposite polarity with minimal transition lengths. Such fields are of interest, for example, for "wavelength shifters," i.e., devices applied to charged particle beams (typically electrons) that produce intense, high photon-energy synchrotron radiation with no net beam steering or displacement.

Figure 3 illustrates the general concept and transition scheme by showing a single quadrupole winding layer split into five sections (FDFDF). The defocusing (D) sections have a reversed axial period, leading to an alternating quadrupole field profile along the length of the bend (for details, see Sec. V). A close-up of the coil transition between a $\mathrm{F}$ and $\mathrm{D}$ section is shown with the current direction at the transition location indicated. This scheme allows the ends of one section to nest inside the next providing an overall compact and efficient approach for the producing an alternating focusing system.

An important practical consideration is the actual conductor reversal. Real conductors have a minimum bend radius, below which internal damage to the superconducting filaments may occur. One characteristic of the CCT concept (for dipole or quadrupole fields) is that the rib thickness, which varies with azimuthal angle, is largest at the pole; the AG-CCT current reversal can occur at the pole, making use of the thick rib in that location. For the conductors envisioned for this application no conductor damage is anticipated from the reversal. Limiting to the pole region does not impact the degrees of freedom available for optics design: the large number of ribs provides a fine discretization of the longitudinal position selection for reversal, and the winding pitch angle provides ultimate fine-tuning if necessary.

\section{ACHROMAT OPTICS OPTIMIZATION FOR A LARGE MOMENTUM ACCEPTANCE GANTRY}

To examine the feasibility of using superconducting AGCCT magnets for locally achromatic curved sections, we want to demonstrate that properly designed, AG-CCT magnets would enable the proton beam to be scanned over a large momentum range $(\Delta p / p> \pm 10 \%)$ while keeping the AG-CCT magnet fields fixed. In order to understand the feasibility, a full gantry optic design was developed that met the constraints listed in Sec. II. The layout of the gantry 


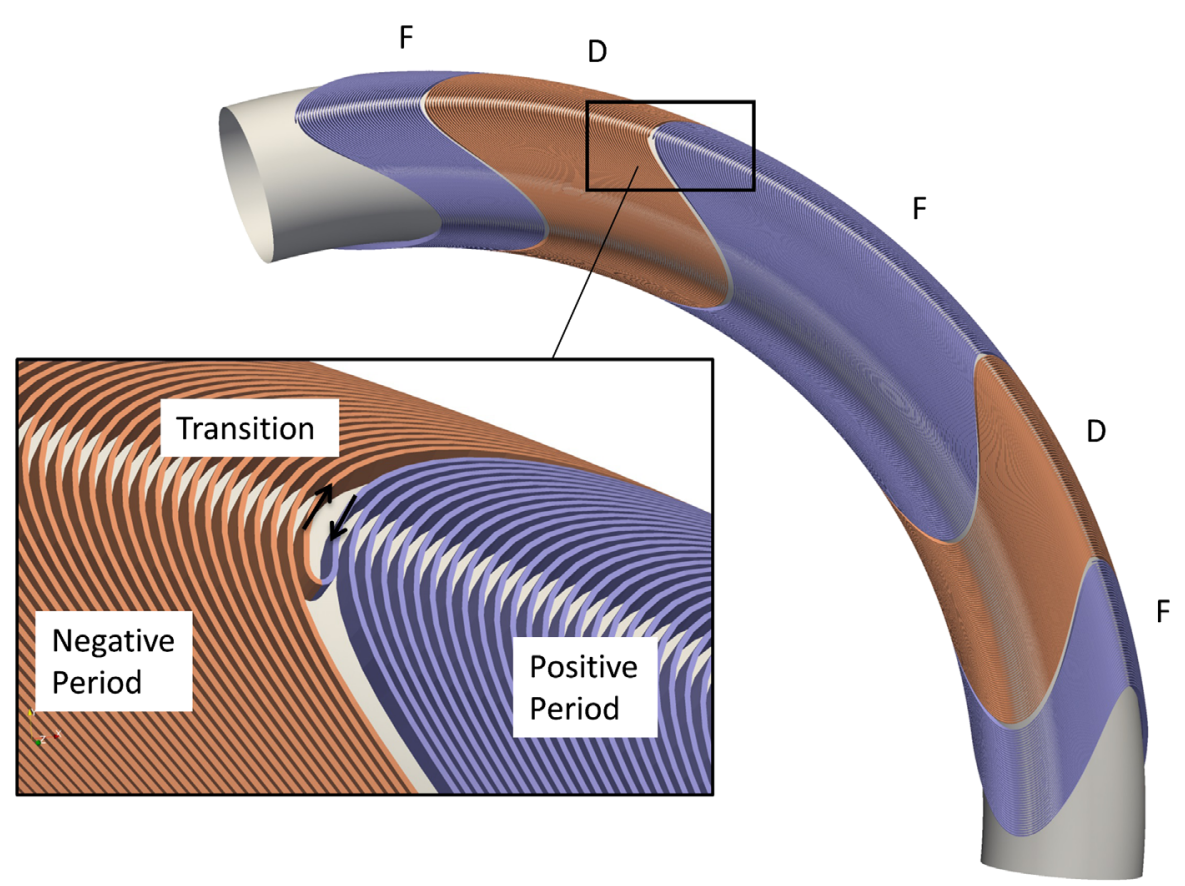

FIG. 3. A single alternating focusing quadrupole layer (corresponding to Layer 1 in Fig. 2) is shown with five sections (FDFDF). The transition scheme between sections of opposite axial period is enlarged with the current direction indicated. This approach allows for the coil to be generated with one continuous winding, where the relative integral strength of the sections can be tuned by adjusting the number and location of the transition points.

is shown in the bottom of Fig. 1. (It is important to note that the goal of the paper is a feasibility demonstration and that the gantry design is not fully optimized.)

In this section, we describe the general approach at arriving at an initial gantry design using a Sharp cutoff fringe field (SCOFF) model. In Sec. V, more details of the AG-CCT magnets are provided as well as an accurate full 3D description of the fields. In Sec. VI we present the simulation results for the gantry using the $3 \mathrm{D}$ AG-CCT fields calculated using the Biot-Savart law derived from the actual AG-CCT coil windings.

The gantry begins at a coupling point (separating the fixed beam line from the gantry). Following the coupling point the beam passes through two locally achromatic, $75^{\circ}$ curved AG-CCT bends labeled B1 and B2. After B2, a collimator is placed at an image of the coupling point defining the beam size and divergence. Following the collimator the beam is transported through a normal conducting quadrupole/octupole matching section consisting of five magnets. The first two, Q1 and Q2, are pure quadrupoles. The second two, QO3 and QO4, are combined function quadrupole/octupole magnets. The final magnet $\mathrm{O} 5$ is a pure octupole. Following the matching section are two sweeper magnets-first an out-of-plane and then an in-plane magnet used to scan the beam transversely over the field. Past the sweeper magnets, the beam passes through a larger aperture, locally achromatic, $90^{\circ}$ curved AG-CCT. Finally the beam will arrive at the isocenter. Dimensions of the gantry are shown in the bottom of Fig. 1.
The length of the gantry is $8.3 \mathrm{~m}$ and the height of the gantry is $2.5 \mathrm{~m}$. (Note that the height is the distance between the isocenter and the center of the beam pipe in the quadrupoles and the length is the distance between the gantry entrance and the isocenter.)

Arriving at such a suitable optics design is a complex multidimensional problem that has to satisfy many and sometimes competing constraints. Here are some of the considerations and approach that went into the design.

\section{A. Considerations and approach in developing the gantry design}

Imagine this gantry divided into three distinct regions: (i) region 1: the beginning of the gantry to the collimator; (ii) region 2: the collimator to the sweeper magnets; and (iii) region 3: the sweeper magnets to the isocenter.

The general approach is to initially treat them separately working backwards from region 3 to region 1 and then iterating.

\section{Region 3}

For region 3, the objective is to scan the beam over a large field in both in- and out-of-plane directions and to scan the beam over a large range of momentum without changing the field of B3.

There are three elements, the two sweeper magnets and the $90^{\circ} \mathrm{AG}-\mathrm{CCT}$. The parameters are the location, size, and field strength of the magnets. Starting with the $90^{\circ}$ 
AG-CCT there are six parameters: (i) distance from the isocenter (1); (ii) aperture (1); (iii) dipole field (1); and (iv) quadrupole field (1) and relative lengths (2) of F and D subsections (assuming a symmetric FDFDF section).

In adjusting these parameters there are a number of considerations. In terms of location we want to place B3 as close to the isocenter as possible while providing sufficient space for nozzle, and patient space around the isocenter. The aperture of B3 needs to be large enough to contain the scanned beam while maintaining reasonable magnetic field values. The dipole field of B3 should be made relatively large for compactness of the gantry. In our case the dipole field of B1, B2, and B3 are adjusted to provide a $1.25 \mathrm{~m}$ bending radius. The quadrupole fields and relative lengths should be adjusted to satisfy the achromat condition $\left(\mathrm{R}_{16}=\mathrm{R}_{26}=0\right)$, and produce a sufficiently large SAD (but not too large to ensure that the beam size and location stays well within the aperture with reasonable strengths).

For the two sweeper magnets there are four parameters: (i) locations (2) and (ii) strengths (2).

In adjusting these parameters there are a number of considerations. It is desirable to locate the sweeper magnets close to B3 for compactness. Also the magnetic field range of the sweeper magnets should be able to rapidly cover a large transverse field.

\section{Region 2}

For region 2, the objective is to image the beam from the collimator to the isocenter over a large range in momentum without changing the fields of B1 and B2. Placing the collimator at the top of the gantry rather than at the beginning of the gantry is advantageous for minimizing the impact of the chromatic aberrations of $\mathrm{B} 1$ and $\mathrm{B} 2$ on the momentum acceptance [7].

One of the challenges in region 2 is transporting a beam with a relatively large emittance $(30 \pi \mathrm{mm}-\mathrm{mrad})$ and imaging it to a small spot size at the isocenter. This results in the matching section having magnets with relatively large apertures, some with strong gradients. The imaging condition will be different as a function of the beam momentum. So these magnets will need to change field as a function of beam momentum or the beam size at the isocenter will change.

For the matching section there are seven magnetic field parameters and five magnet locations: (i) quadrupole gradients (4); (ii) octupole gradients (3); and (iii) locations (5) of the quadrupoles and octupoles.

In adjusting these parameters there are a number of considerations. For the locations the main consideration is compactness. The quadrupole fields are adjusted to match the beam from the collimator to the isocenter resulting in four linear constraints: (i) imaging: $R_{12}=R_{34}=0$ and (ii) magnification: $R_{11}=R_{33}=C$. Note that $C$ remains unchanged over the range of the beam momentum.
In the optics chosen here the magnification at the isocenter is 1.9 with the horizontal and vertical phase space at the collimator assumed to be $3 \mathrm{~mm} \times 10 \mathrm{mrad}$. The octupole fields are used to minimize nonlinear phase space distortion particularly $\mathrm{U}_{1222}=\mathrm{U}_{3444}=\mathrm{U}_{1244}=0$. These are the three main third order spherical aberration terms.

\section{Region 1}

For region 1, the goals are an achromatic system from the coupling point of the collimator to the collimator. This is done with two identical $75^{\circ}$ locally achromatic AG-CCT magnets. For the matching there are six parameters: (i) locations (1); (ii) aperture (1); (iii) dipole field (1); and (iv) quadrupole field (1) and relative lengths (2) (of the F and D sections assuming a symmetric FDFDF section).

In adjusting these parameters there are a number of considerations. The height needs to be the same as region 3 . There is also a need to image the beam from the beginning of the gantry to the collimator. In this design the imaging is 1 to 1 . Both $\mathrm{B} 1$ and $\mathrm{B} 2$ are assumed to be symmetric and locally achromatic $\left(\mathrm{R}_{16}=\mathrm{R}_{26}=0\right)$. They are also identical to each other, just bending in opposite directions.

\section{B. Gantry optics}

As mentioned earlier, a SCOFF model was used for the magnets when initially designing the linear gantry optics. This permits faster linear optimization and checking of some achromatic and kinematic effects. After optimization the effects of the full field of the AG-CCT were used for simulation (the results of the beam dynamics with the full model is shown in Sec. VI).

\section{SCOFF model for the AG-CCT}

The SCOFF model for the AG-CCT the fields are shown for the $90^{\circ}$ and $75^{\circ}$ magnets in Figs. 11 and 12. One thing to notice is that the magnitude of the gradient for each of the FDFDF quadrupole sections is the same-only the sign changes. Since the magnet is symmetric and the total bending angle is fixed there are just three parameters.

TABLE II. Properties of the $90^{\circ}$ and $75^{\circ}$ AG-CCT magnets (SCOFF model).

\begin{tabular}{lcc}
\hline \hline & $75^{\circ}(\mathrm{B} 1, \mathrm{~B} 2)$ & $90^{\circ}(\mathrm{B} 3)$ \\
\hline Bore radius $(\mathrm{mm})$ & 50 & 150 \\
Bending radius $(\mathrm{m})$ & 1.25 & 1.25 \\
Gradient $(\mathrm{T} / \mathrm{m})$ at $217 \mathrm{MeV}$ & 48.74 & 17.43 \\
F Angle (degree) & 13.38 & 10.02 \\
D Angle (degree) & 15.80 & 17.79 \\
F Angle (degree) & 16.63 & 34.39 \\
D Angle (degree) & 15.80 & 17.79 \\
F Angle (degree) & 13.38 & 10.02 \\
\hline \hline
\end{tabular}


TABLE III. Properties of the normal conducting resistive magnets.

\begin{tabular}{lcrccc}
\hline \hline & Q1 & \multicolumn{1}{c}{ Q2 } & QO3 & QO4 & O5 \\
\hline Radial aperture $(\mathrm{mm})$ & 12.5 & 50 & 80 & 60 & 50 \\
Length $(\mathrm{m})$ & 0.25 & 0.5 & 0.5 & 0.25 & 0.1 \\
Gradient $(\mathrm{T} / \mathrm{m})^{\mathrm{a}}$ & 7.55 & 18.0 & -12.1 & 16.2 & 0.0 \\
Octupole $\left(\mathrm{T} / \mathrm{m}^{3}\right)^{\mathrm{a}}$ & 0.0 & 0.0 & -17.8 & 426 & -1069 \\
\hline \hline
\end{tabular}

${ }^{\mathrm{a}}$ Note that the quadrupole and octupole strengths are peak values at $217 \mathrm{MeV}$.

[These are the magnitude of the gradient (1), the locations of the transition from the first $\mathrm{F}$ to the first D (1), and the transition from the first $\mathrm{D}$ to the second $\mathrm{F}(1)$.] These three parameters are adjusted to satisfy the achromatic condition and to provide good transport properties. In Table II the gradients and angular lengths for the $90^{\circ}$ and $75^{\circ}$ magnets are given. Later they are used as a first guess at the current and angular lengths for the full AG-CCT model. Then in the real model they are adjusted to restore in great part the linear properties of the SCOFF model.

\section{Normal conducting matching section}

The values of the normal matching section quadrupoles and octupoles are provided in Table III. The gradients are all such that the pole tip field is kept below 1.2 Tesla. As can be seen in Table III, the apertures are not all the same. In particular, the aperture for QO3 is larger to accommodate the larger vertical beam size in that quadrupole (determined by the red ray in Fig. 5).

\section{Principle rays for the full gantry}

In Figs. 4 and 5 the principle rays are shown using a SCOFF model for the full gantry. The beam is round (identical for both in- and out-of-plane directions) at the entrance of the gantry with a phase space that is $3 \mathrm{~mm} \times 10 \mathrm{mrad}$. The starting conditions for the principle rays are $3 \mathrm{~mm}, 10 \mathrm{mrad}$ and $\Delta p / p=10 \%$ in-plane (see Fig. 4) and $3 \mathrm{~mm}$ and $10 \mathrm{mrad}$ out-of-plane (see Fig. 5).

From Fig. 4 one sees that to first order each bend (B1, $\mathrm{B} 2$, and B3) is locally achromatic. Also there is a 1 to 1 image of the beam from the entrance of the gantry to the collimator. There is a 1:1.9 image of the beam from the collimator to the isocenter, which fulfills the requirement to have the beam width to be around $6 \mathrm{~mm}$. Note that B1 and B2 form an optical system that is both imaging and telescopic. The additional symmetry helps to eliminate many aberrations.

\section{Sweeper magnets to the isocenter}

Figures 6 and 7 show the in- and out-of-plane rays from the sweeper magnet to the isocenter. The initial conditions for the rays plotted are 25 and 30 mrad for the out-of-plane and in-plane kickers, respectively. One sees that in the linear approximation this results in an offset at the isocenter of $14.1 \mathrm{~cm}$ out-of-plane and $13.3 \mathrm{~cm}$ in-plane, respectively.

\section{Summary of linear gantry properties}

In Table IV we present a number of the beam and design gantry properties as determined by the simple SCOFF model. As one can see all satisfy the comparable constraints outlined in Table I. So it is a good baseline for a design.

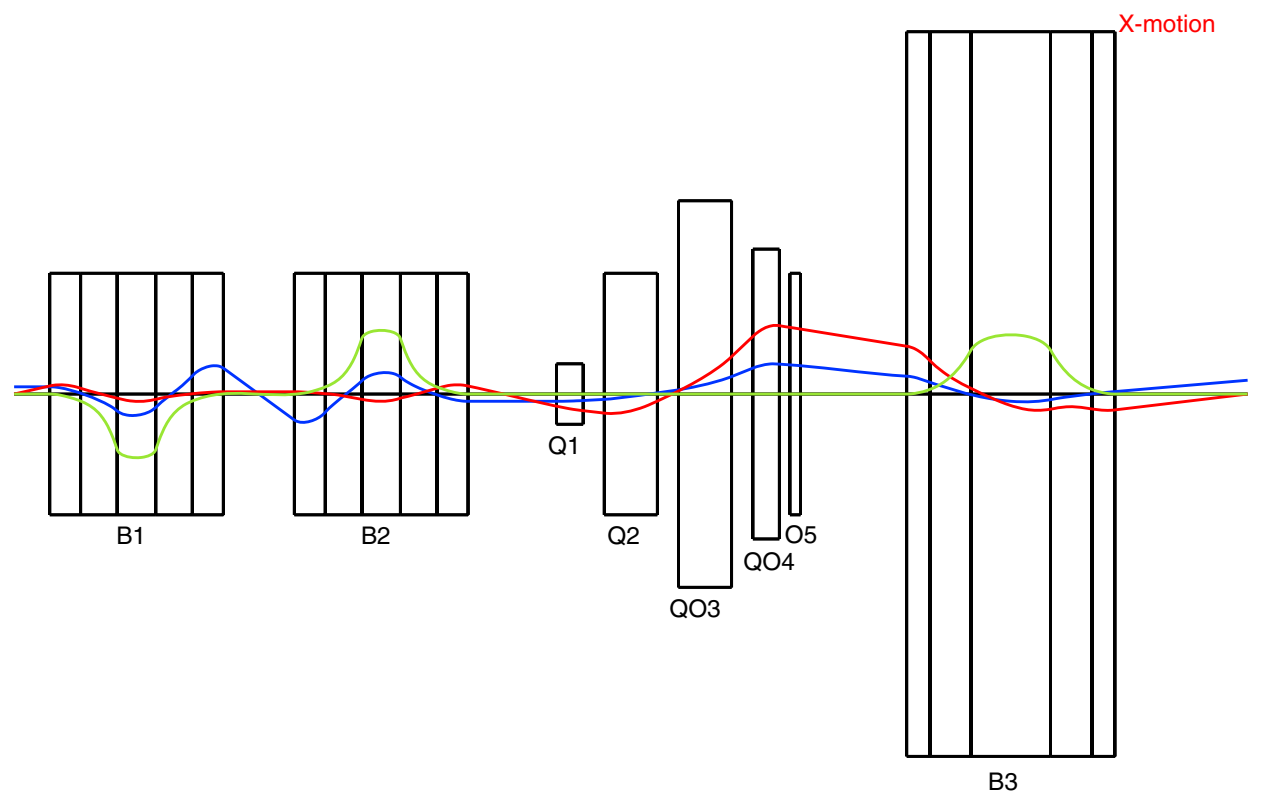

FIG. 4. Principle in-plane rays for the full system [initial rays are $x=3 \mathrm{~mm}$ (blue), $p_{x}=10 \mathrm{mrad}(\mathrm{red})$, and $\left.\Delta p / p=10 \%(\mathrm{green})\right]$. The full width of B1, B2 and B3 are 10,10 and $30 \mathrm{~cm}$, respectively. 


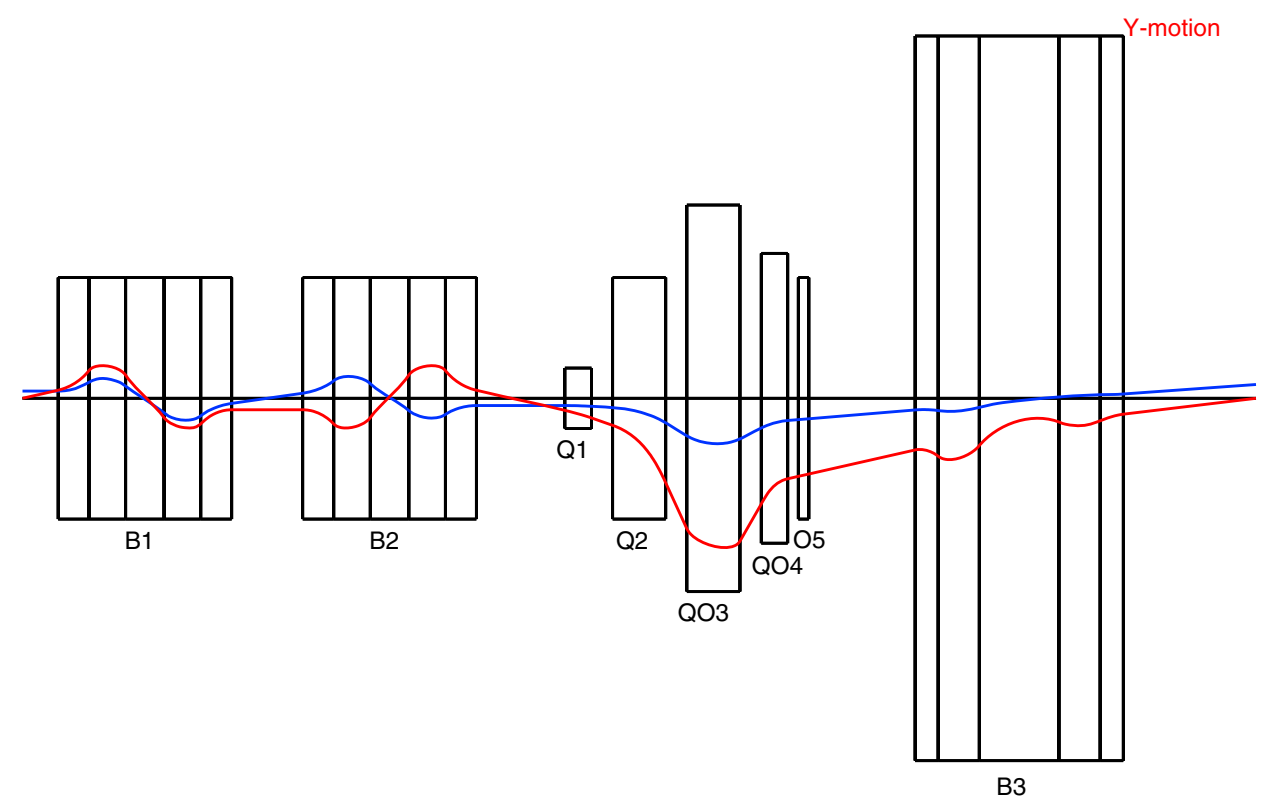

FIG. 5. Principle out-of-plane rays for the full system [initial rays are $y=3 \mathrm{~mm}$ (blue), and $p_{y}=10 \mathrm{mrad}$ (red)]. The full width of $\mathrm{B} 1, \mathrm{~B} 2$, and $\mathrm{B} 3$ are 10,10 , and $30 \mathrm{~cm}$, respectively.

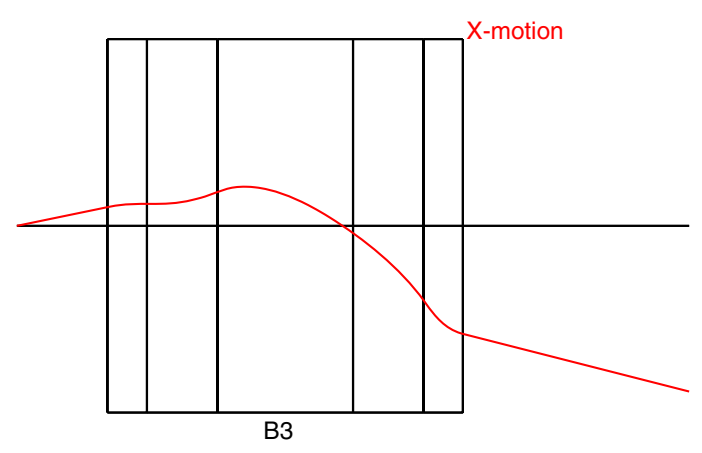

FIG. 6. In-plane ray with an initial angle $30 \mathrm{mrad}$ from sweeper corresponding to an offset of $13.3 \mathrm{~cm}$ at the isocenter.

In Sec. V the properties of the B1, B2, and B3 AG-CCTs will be discussed. This is followed in Sec. VI by a simulation of the gantry using the fields of the coils of the AG-CCTs (to see what the actual scanning volume and beam distortion will be).

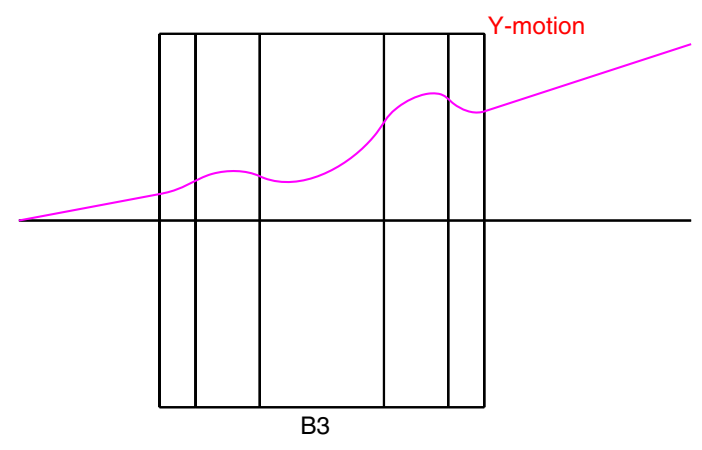

FIG. 7. Out-of-plane ray with an initial angle of $25 \mathrm{mad}$ from sweeper corresponding to an offset of $14.1 \mathrm{~cm}$ at the isocenter.

\section{AN AG-CCT MAGNET DESIGN ADDRESSING THE OPTIMAL OPTIC DESIGN}

A preliminary magnet design study has been performed to verify the feasibility of using the AG-CCT concept for the gantry optics presented in Sec. IV. A four layer design was considered for both the 75 degree $(\mathrm{B} 1, \mathrm{~B} 2)$ and 90 degree bending (B3) sections. Each bending section contains two AG-CCT quadrupole layers nested inside two outer CCT dipole layers. The conductor is positioned in grooved winding mandrels that also provide structural support. The winding mandrel parameters are shown in Table V, where the channel depth is determined by the number of strands per layer.

TABLE IV. Beam and linear gantry properties calculated from SCOFF model.

\begin{tabular}{|c|c|}
\hline Emittance $^{\mathrm{a}}$ & $30 \pi \mathrm{mm}-\mathrm{mrad}$ \\
\hline Beam size at entrance $^{b}$ & $3 \mathrm{~mm}$ \\
\hline Beam divergence at entrance $^{b}$ & $10 \mathrm{mrad}$ \\
\hline Beam size at collimator $^{b}$ & $3 \mathrm{~mm}$ \\
\hline Beam divergence at collimator $^{\mathrm{b}}$ & $10 \mathrm{mrad}$ \\
\hline Beam size at isocenter ${ }^{\mathrm{b}}$ & $5.7 \mathrm{~mm}$ \\
\hline Beam divergence at isocenter $^{b}$ & $5.26 \mathrm{mrad}$ \\
\hline Beam energy range $(\mathrm{MeV})$ & 70 to 220 \\
\hline In-plane SAD & $3.6 \mathrm{~m}$ \\
\hline Out-of-plane SAD & $3.3 \mathrm{~m}$ \\
\hline Space from the end of $\mathrm{B} 3$ to the isocenter & $1.25 \mathrm{~m}$ \\
\hline Maximum sweeper angle & $\pm 30 \mathrm{mrad}$ \\
\hline In-plane sweeper response at isocenter & $4.4 \mathrm{~mm} / \mathrm{mrad}$ \\
\hline Out-of-plane sweeper response at isocenter & $5.7 \mathrm{~mm} / \mathrm{mrad}$ \\
\hline
\end{tabular}

${ }^{\mathrm{a}}$ Emittance is the same for in-plane and out-of-plane directions. ${ }^{\mathrm{b}}$ The beam is round at this location. 
TABLE V. Winding Mandrel geometry (in mm).

\begin{tabular}{|c|c|c|c|c|c|c|c|c|c|}
\hline \multirow[b]{2}{*}{ Lay } & \multirow[b]{2}{*}{ Type } & \multicolumn{4}{|c|}{75 degree $(\mathrm{B} 1, \mathrm{~B} 2)$} & \multicolumn{4}{|c|}{90 degree (B3) } \\
\hline & & $\mathrm{Ri}$ & Ro & Wall thickness & Channel & $\mathrm{Ri}$ & Ro & Wall thickness & Channel \\
\hline 1 & AG-CCT & 50 & 64.3 & 14.3 & $2.0 / 11.3$ & 150 & 169 & 19.0 & $2.0 / 16.0$ \\
\hline 2 & AG-CCT & 64.3 & 78.6 & 14.3 & $2.0 / 11.3$ & 169 & 188 & 19.0 & $2.0 / 16.0$ \\
\hline 3 & CCT dipole & 78.6 & 85.1 & 6.5 & $2.0 / 3.5$ & 188 & 196.2 & 8.2 & $2.0 / 5.2$ \\
\hline 4 & CCT dipole & 85.1 & 91.6 & 6.5 & $2.0 / 3.5$ & 196.2 & 204.4 & 8.2 & $2.0 / 5.2$ \\
\hline
\end{tabular}

\section{A. Magnetics}

The magnetic layout is initially developed using the SCOFF model, where each magnetic element is defined in terms of traditional dipole, quadrupole, etc. multipole terms of finite length, with no end effects (i.e., sharp cutoff fields). The SCOFF model here is not optimized, but is rather used as a starting point for the magnetic design optimization. The result of the SCOFF model effort is an initial baseline design that includes parameters such as bore diameter and integrated harmonic strength.

The CCT models (both dipole and quadrupole) are then designed so as to approach the SCOFF model in terms of integrated multipole strengths. Key parameters in the CCT model include conductor dimensions, spare thickness, and $\mathrm{CCT}$ winding pitch. Both the dipole and the quadrupole layers are designed in pairs; in each pair the winding is designed so as to cancel the solenoidal field contribution, while adding the multipole contribution. To increase the strength of the dipole, for example, one can increase the conductor radial size or increase the number of dipole layers (e.g., 4 instead of 2).

The CCT concept provides numerous degrees of freedom for design optimization. In the case of a straight CCT dipole design, the tilt angle of the windings at the midplane is chosen to maximize the integrated dipole field for a given physical length and bore diameter. For typical high field accelerator dipoles this angle is between 15 and 30 degrees. The resulting field is very close to a perfect dipole, as described in [25]. A similar approach of simply canting the windings to produce a dipole, when applied in the curved geometry of a gantry magnet, leads to the generation of a "dipole" plus higher order "harmonics" (quadrupole, sexupole, etc.). Note that due to the curved geometry of the gantry magnets, the traditional 2D field expansion in terms of cylindrical multipoles, e.g., dipole, quadrupole, sextupole, etc. is not formally valid, since the fields are three dimensional. In a toroidal coordinate system a 2D field expansion can be written, but that is beyond the scope of this paper. Here we continue to use the traditional nomenclature to guide the initial design process, but an important aspect of our approach is to use actual fields, evaluated via Biot-Savart integration of the conductor currents, in the tracking simulations and for the generation of field maps so that no approximations are made in the field-optics interface.
Eliminating the curvature-induced higher order multipoles requires the optimization of a set of windings to produce a cross section that is left-right asymmetric. The approach taken for this study was to parametrize the coil path with a set of winding coefficients that relate the two toroidal angles such that,

$$
\begin{aligned}
\eta & =\eta_{0} \\
\xi & =\xi \\
\phi(\xi) & =C_{n} \sin (n \xi)+\frac{\phi_{0}}{2 \pi} \xi,
\end{aligned}
$$

where $(\eta, \xi, \phi)$ are the toroidal coordinates, $\phi_{0}$ is the angular pitch of the winding, $\eta_{0}$ restricts the path to the surface of a torus, and $0 \leq \xi \leq 2 \pi k$ produces $k$ winding turns. The detailed winding pattern associated with this parametrization can be readily visualized by transforming to Cartesian coordinates. (For more details, see [8].) The winding coefficients $C_{n}$, which determine the degree of axial modulation of the path, were numerically optimized for each pair of layers with the figure of merit being a local cylindrical expansion of the fields on a reference radius from the center of the bore compared to the desired SCOFF model harmonics.

The result of this process for the dipole and quadrupole layers of the 75 degree bend $(\mathrm{B} 1, \mathrm{~B} 2)$ is shown in Fig. 8. Similar to a straight CCT it is seen that the axial modulation of the tilted path can be used to create single higher order harmonics. For this application each of the sets of layers are optimized to produce a single harmonic, but the process can also be applied to produce the combined function fields as described in $[8,18]$.

In this study we have used the Superconducting Super Collider (SSC) inner NbTi strand as the basis for analysis, as the conductor (a) has been fully developed and optimized for large scale use, (b) is very well defined and its properties well established, and (c) it is readily available commercially. In case of the 75 degree bend, the 8 conductors in the two dipole layers will be powered in series, thereby allowing for relatively low operating current for the magnet system; similarly the 26 conductors in the two quadrupole layers will be powered in series from a second power supply. Fig. 9 shows the loading curves of the 75 degree bend. Similar arrangement is done for the 90 degree bend; details are shown in Fig. 10. 

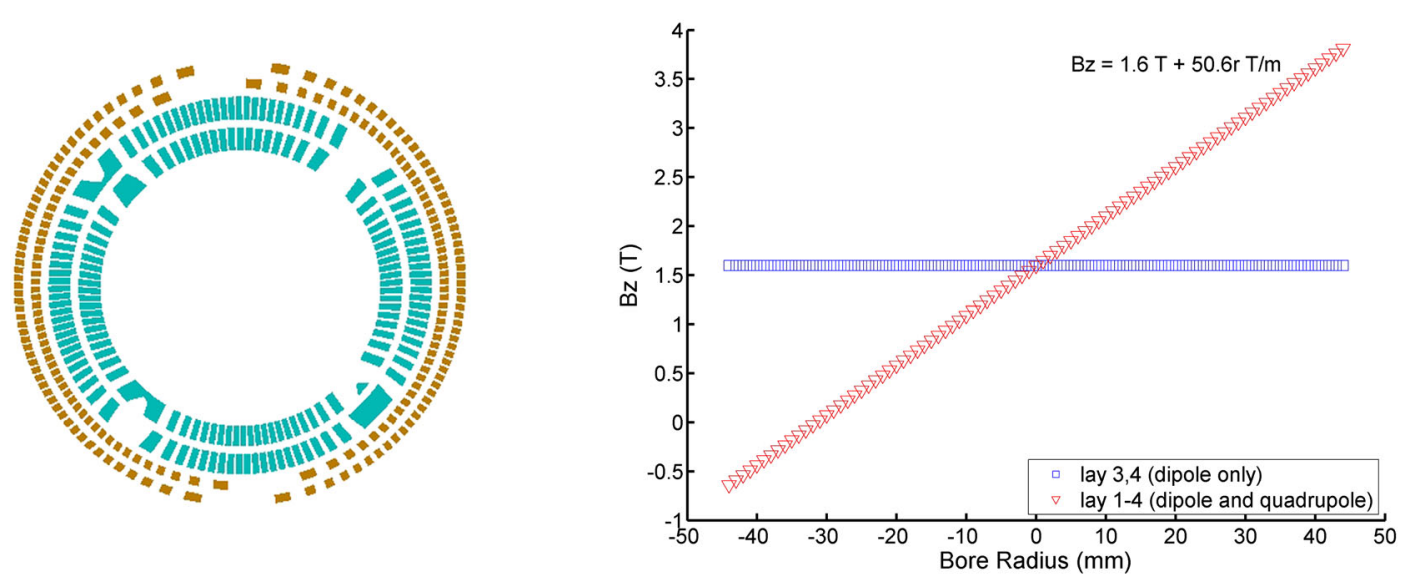

FIG. 8. A cross section of the combined magnet system conductor positions is shown on the left for the 75 degree bend. The dipole windings (outer two layers) are designed to provide a constant $B_{z}(\rho)$ field profile in a transverse slice (i.e., $\phi=$ constant). The quadrupole windings (inner two layers) are designed to provide a quadrupole field in a transverse slice, with zero field at the center of the aperture. This can be seen in the right-hand figure showing the vertical field on the midplane produced by the windings.

This approach is motivated by the need to keep the current low in order to be compatible with a cryocooler-based cryogenic system. The detailed design of a cable with these properties is beyond the scope of this paper. The conductor properties are provided in Table VII, and cable properties are provided in Table VIII. The corresponding dimensions of the channels in the mandrels are provided in Table V.

Alternative superconductors exist that can be considered for this application. Examples include the low-temperature superconductors (LTS) $\mathrm{Nb}_{3} \mathrm{Sn}$ and $\mathrm{MgB}_{2}$, and hightemperature superconductors (HTS) $\mathrm{Bi}_{2} \mathrm{Sr}_{2} \mathrm{CaCu}_{2} \mathrm{O}_{8+x}$ and $\mathrm{YBa}_{2} \mathrm{Cu}_{3} \mathrm{O}_{7-\delta}$ (see Table VI for material property comparison). The LTS conductor $\mathrm{Nb}_{3} \mathrm{Sn}$ is an inter metallic compound created through a heat treatment at $\sim 640 \mathrm{C}$ in

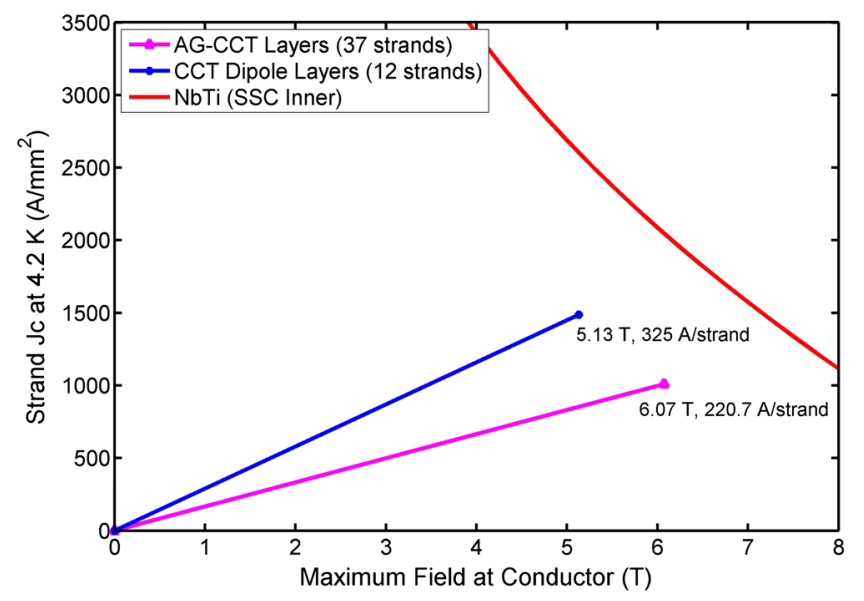

FIG. 9. The short sample in the superconductor using SSC inner NbTi strand is shown for both the AG-CCT and CCT dipole layers of the 90 degree bend (B3). Both sets of layers have a current margin of more than $20 \%$ for the given number of strands. The full details can be found in Table VIII. an inert gas environment. After the heat treatment the conductor is brittle, hence the material is often used in a "Wind and React" (W\&R) manner. $\mathrm{Nb}_{3} \mathrm{Sn}$ is routinely used for high field application, where NbTi is no longer superconducting, but is rarely used for low field applications due to the added complications associated with the heat treatment. For this application the field does not justify the use of $\mathrm{Nb}_{3} \mathrm{Sn}$; however the material has a significantly higher critical temperature, which is very appealing for cryocooled systems where temperature gradients may result in higher conductor temperatures.

$\mathrm{MgB}_{2}$ is a recently developed superconductor that holds promise for applications such as this due to its relatively high $\mathrm{T}_{c}(\sim 18 \mathrm{~K})$, isotropic properties, and potentially low cost. At this time, however, its transport current density at

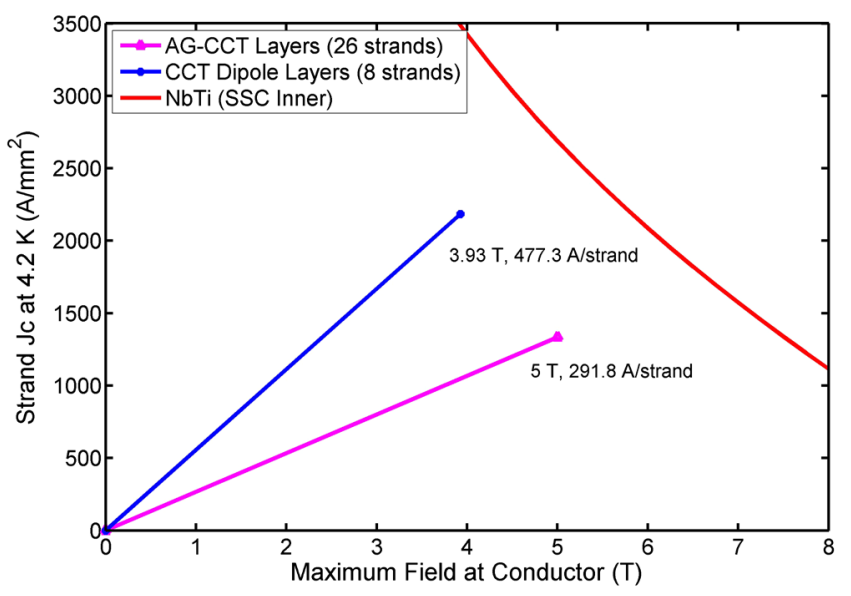

FIG. 10. The short sample in the superconductor using SSC inner NbTi strand is shown for both the AG-CCT and CCT dipole layers of the 75 degree bend $(\mathrm{B} 1, \mathrm{~B} 2)$. Both sets of layers have a current margin of more than $25 \%$ for the given number of strands. The full details can be found in Table VIII. 
TABLE VI. Low and high temperature superconductor parameters.

\begin{tabular}{lccccl}
\hline \hline Superconductor & $T_{c}[\mathrm{~K}]$ & $B_{c 2}[\mathrm{~T}]$ & $J_{E}(5 \mathrm{~T}, 4.2 \mathrm{~K})\left[\mathrm{A} / \mathrm{mm}^{2}\right]$ & Process & Comments \\
\hline $\mathrm{NbTi}$ & $8.9-9.3$ & $10.5-11$ & $\sim 1000$ & Wind & Standard material for such applications \\
$\mathrm{Nb}_{3} \mathrm{Sn}$ & $17-23$ & $19-27$ & $\sim 1500$ & W \& R & Well established but fab complexity \\
$\mathrm{MgB}_{2}$ & $\sim 39$ & $9-10$ & $\sim 200$ & W/W\&R & More competitive at 3 T and below \\
$\mathrm{Bi}_{2} \mathrm{Sr}_{2} \mathrm{CaCu}_{2} \mathrm{O}_{8+x}$ & $70-75$ & $\sim 110$ & $\sim 600$ & W\&R & R\&D, fab complexity \\
$\mathrm{YBa}_{2} \mathrm{Cu}_{3} \mathrm{O}_{7-\delta}$ & $92-95$ & $\sim 110$ & $\sim 900$ & Wind & Anisotropic, bend limitations \\
\hline \hline
\end{tabular}

TABLE VII. SSC inner NbTi strand.

\begin{tabular}{lc}
\hline \hline Diameter & $0.8 \mathrm{~mm}$ \\
$\mathrm{Jc}(5 \mathrm{~T}, 4.2 \mathrm{~K})$ & $2750 \mathrm{~A} / \mathrm{mm}^{2}$ \\
$\mathrm{Cu} / \mathrm{SC}$ & 1.3 \\
Filament $\phi$ & $6.0 \mu \mathrm{m}$ \\
\hline \hline
\end{tabular}

4-5 $\mathrm{T}$ is not very competitive. A summary of superconductor options is given in Table VI.

\section{B. Structure}

The magnetic models used for tracking are directly linked to CAD models of the coils and structures. The mechanical analysis process developed in [26] can be applied to the gantry magnet discussed here. Although such a study is beyond the scope of this paper, we have performed some preliminary analysis to provide an estimate of the weight of the cold mass structure. The mandrels/spars, superconducting material, and aluminum support tube that prestresses the system upon cooldown, weigh in total about 500 to $750 \mathrm{~kg}$. Based on this study we estimate that the complete 90 degree bend magnet with internal support structure, cryogenic shielding, cryostat vacuum vessel, and cryocoolers, will likely weigh on the order of 2000 to $3000 \mathrm{~kg}$. This is a very significant reduction (more than a factor of 10 over the 48 ton PSI Gantry-II final magnet) over existing technology, and we anticipate that this will have important implications for cost and stability of the rotating support structures. Here we assume that iron shielding for stray fields is provided external to the cryostat and is not included in this weight.

\section{Cooling}

The preferred cooling method for a rotating superconducting gantry is via conduction, using cryocoolers to provide the requisite cooling power. Such an approach eliminates the need for liquid helium and the associated safety and maintenance issues when these are mounted on a rotating gantry. The cryocoolers can be aligned with the rotation axis of the gantry to eliminate any variation in the cooling power with rotation, and the cold-heads can be connected in parallel to various parts of the magnet system using high-conductivity links. The gantry design described here dramatically reduces the need to ramp the superconducting magnets for a tumor treatment.

\section{SIMULATION PROCESS AND RESULTS OF AN AG-CCT GANTRY}

In this section we present the results of the beam dynamics simulations. There are two objectives of the simulations: (i) Quantify the size of the 3D (transverse and momentum) volume that the proton beam can be scanned while keeping the magnetic fields of the AG-CCT fixed. (ii) Determine the degree to which the beam distorts while being scanned over that 3D volume.

The size of the $3 \mathrm{D}$ volume as well as the beam distortion is determined in large part by nonlinearities of the beam motion. The nonlinear beam motion is in turn determined by the nonlinearities in the magnetic fields as well as nonlinearities in the equations of motion. Therefore it is essential to simulate the beam motion as precisely as possible - using accurate field representations and minimizing assumptions about the equations of motion. The latter is sometimes not fully appreciated. For instance many simulation codes use assumptions, such as a paraxial approximation, that break down for cases like ours where the emittance is large or where the beam is being scanned over large angles. Therefore in our simulations, great lengths were made not only to model the fields precisely but also to minimize assumptions in the equations of motion. In this section we first list the assumptions made

TABLE VIII. Short sample at highest magnet current.

\begin{tabular}{|c|c|c|c|c|c|c|c|c|c|}
\hline \multirow[b]{2}{*}{ Lay } & \multirow[b]{2}{*}{ Type } & \multicolumn{4}{|c|}{75 degree $(\mathrm{B} 1, \mathrm{~B} 2)$} & \multicolumn{4}{|c|}{90 degree bore (B3) } \\
\hline & & $\mathrm{I} /$ strand $(\mathrm{A})$ & $B_{\text {cond }} \cdot(\mathrm{T})$ & Strands & Margin (\%) & I/strand (A) & $B_{\text {cond }} \cdot(\mathrm{T})$ & Strands & Margin (\%) \\
\hline 1 & AG-CCT & 291.8 & 5.0 & 26 & 24 & 220.7 & 6.03 & 37 & 21 \\
\hline 1 & AG-CCT & 291.8 & 5.0 & 26 & 24 & 220.7 & 6.07 & 37 & 21 \\
\hline 3 & CCT dipole & 477.3 & 3.93 & 8 & 26 & 325 & 5.13 & 12 & 26 \\
\hline 4 & CCT dipole & 477.3 & 2.86 & 8 & 48 & 325 & 4.03 & 12 & 45 \\
\hline
\end{tabular}


about the fields and about the equations of motion. Next we discuss the simulation and analysis process. Then the results are presented followed by some discussion points.

\section{A. Magnetic field model and equations of motion}

The magnetic field models of the different elements are presented here. For the AG-CCT, the model used is described in Sec. V. In the model there is no iron so the 3D magnetic fields are derived from the coil placements and currents using the Biot-Savart law. For the other magnets less effort was made to detail their design. Here is a summary of the field models for the AG-CCTs, resistive quadrupoles, octupoles, and sweeper magnets: (i) AG-CCT magnets: at each position the 3D fields are calculated using Biot-Savart Law derived from about 200,000 line current segments of the two dipole and two quadrupole coils; (ii) resistive quadrupoles: soft edge fringe field model; (iii) resistive octupoles: soft edge fringe field model; and (iv) sweeper magnets: thin dipole kick.

The equations of motion were evaluated using the simulation code COSY Infinity [27]. All relativistic effects are included and there is no paraxial approximation. The integration method is a seventh order Runge-Kutta integrator with adaptive step size control [28]. A powerful feature of COSY Infinity is the ability to generate a nonlinear transfer map of arbitrarily high order. This transfer map is a polynomial expansion describing how particles with given initial transverse positions and momenta, and energy at one location in the gantry is mapped to another downstream. The transfer map is generated using the enclosed differential algebra (DA) package. The map is valuable for a number of reasons, including: adjusting the magnet strengths to produce the proper optical properties, interpreting the tracking results, and for fast particle "map" tracking.

\section{B. Process}

The general process followed to prepare and then perform the simulation is the following: (i) full field model of the AG-CCT is adjusted to be as close as possible to the linear SCOFF model (see Sec. VIB 1); (ii) AG-CCT is included into the full gantry and the quadrupoles and octupoles are adjusted (see Sec. VI B 2): (a) quadrupoles adjusted to get the proper design imaging condition from the collimator to the isocenter; (b) octupoles are set correct spherical aberrations in order to minimize phase space distortion; (iii) particles are tracked from the collimator to the isocenter.

\section{Fitting the AG-CCT}

The process for fitting the SCOFF model is the following: (i) Begin with the SCOFF model of the AG-CCTs, where the following quantities are determined: (a) angles for the transition and (b) magnitude of the FDFDF quadrupole gradients. (ii) Use these as a first guess for the full field
TABLE IX. AG-CCT split.

\begin{tabular}{|c|c|c|c|c|c|c|}
\hline \multirow[b]{2}{*}{ Type } & \multicolumn{3}{|c|}{75 degree $(\mathrm{B} 1, \mathrm{~B} 2)$} & \multicolumn{3}{|c|}{90 degree (B3) } \\
\hline & nturns & $\begin{array}{l}\text { Bend } \\
\text { (deg) }\end{array}$ & $\begin{array}{c}\text { Gradient } \\
(\mathrm{T} / \mathrm{m})\end{array}$ & nturns & $\begin{array}{l}\text { Bend } \\
\text { (deg) }\end{array}$ & $\begin{array}{c}\text { Gradient } \\
(\mathrm{T} / \mathrm{m})\end{array}$ \\
\hline $\mathrm{F}$ & 62 & 13.4 & 50.6 & 44 & 10.3 & 19.7 \\
\hline $\mathrm{D}$ & 73 & 15.8 & -50.6 & 79 & 18.6 & -19.7 \\
\hline $\mathrm{F}$ & 77 & 16.6 & 50.6 & 137 & 32.2 & 19.7 \\
\hline $\mathrm{D}$ & 73 & 15.8 & -50.6 & 79 & 18.6 & -19.7 \\
\hline $\mathrm{F}$ & 62 & 13.4 & 50.6 & 44 & 10.3 & 19.7 \\
\hline Total & 347 & 75 & & 383 & 90 & \\
\hline
\end{tabular}

model of the AG-CCT: (a) angles adjusted to the nearest integer winding number and (b) quadruple are adjusted to obtain similar linear properties. (iii) Track through the full field model of the AG-CCTs using COSY and DA determining the linear transfer matrix. (iv) Adjust the current to minimize the difference between the linear matrix calculated here and the linear matrix of the SCOFF model. The result is shown in Table IX.

The resulting fields are shown in Figs. 11 and 12 and can be compared with SCOFF model fields used (dotted lines) in the same figures. There are several interesting observations. The first is that for the real fields, the fringes and transitions between the various $\mathrm{F}$ and $\mathrm{D}$ sections are smooth and well behaved. A second observation is that the agreement between the real and SCOFF fields is closer in the 75 degree AG-CCT than in the 90 AG-CCT. This can be easily understood because the $75^{\circ}$ magnet has a much smaller $(3 \times)$ aperture compared with the $90^{\circ}$ degree magnet. Nevertheless we see in the next sections that from a linear optics and beam dynamic point of view both the $90^{\circ}$ and $75^{\circ}$ sections are well behaved.

\section{Adjusting the matching section}

Compared to the SCOFF model, the full field AG-CCT fields change the SAD slightly (the in-plane SAD is $8.4 \%$

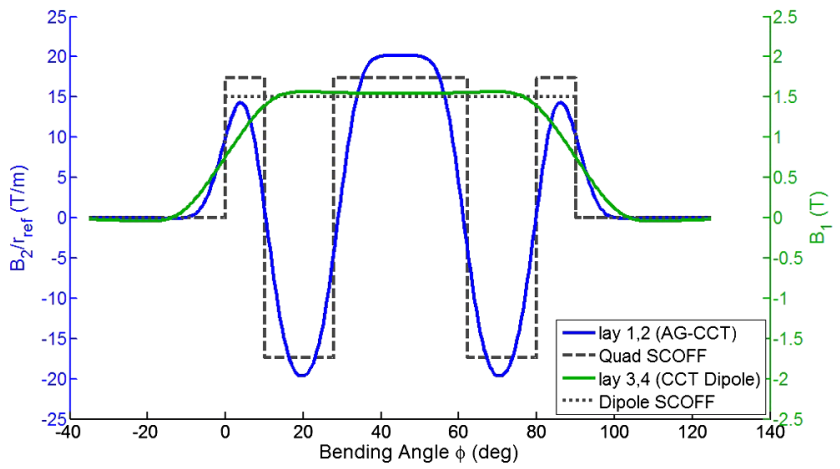

FIG. 11. The dipole $\left(\mathrm{B}_{1}\right)$ and alternating quadrupole $\left(\mathrm{B}_{2} / r_{\text {ref }}\right)$ contributions from the CCT dipole and AG-CCT layers are shown as a function of bending angle for the 90 degree bend (B3). Note that $r_{\text {ref }}$ is the bore radius which is listed in Table II. 


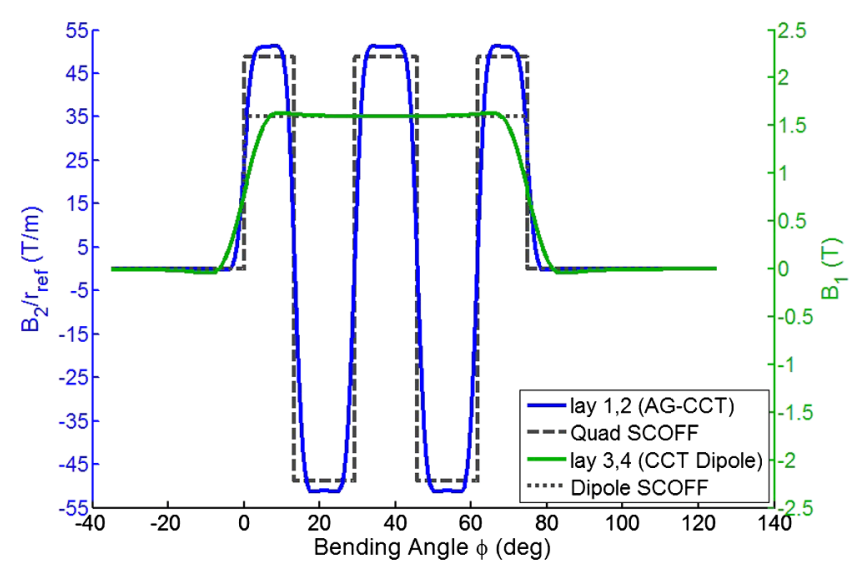

FIG. 12. The dipole $\left(\mathrm{B}_{1}\right)$ and alternating quadrupole $\left(\mathrm{B}_{2} / r_{\text {ref }}\right)$ contributions from the CCT dipole and AG-CCT layers are shown as a function of bending angle for the 75 degree bend (B1, B2). Note that $r_{\text {ref }}$ is the bore radius which is listed in Table II.

longer and the out-of-plane SAD is $4.6 \%$ shorter). Also for the full fields, the in-plane sweeper response at the isocenter is $10.3 \%$ stronger and the out of plane is $7.5 \%$ weaker. As a result, the quadrupoles, in the matching section, need to be adjusted to compensate for the difference. While the weakest quadrupole (Q1) changes significantly (40\%), the other three quadrupoles, Q2, Q3, and Q4, only change $5 \%, 4 \%$ and $2.4 \%$ in the quadrupole gradients, respectively. Furthermore, the principle rays behave very similarly. There still remains a small uncompensated difference due to a finite coupling term resulting from the different coils being at slightly different radius in the AG-CCT.

Once the linear lattice is fixed the next step is to adjust the octupoles to minimize spherical distortions. Such distortions are caused by several things: (i) nonlinearities in the magnetic fields coming from either the body or fringes of the magnets and (ii) nonlinear kinematic effects.

\section{Adjusting $B 1$ and $B 2$}

The process for adjusting B1 and B2 is very similar to the process used for B3. Beginning with the SCOFF model, determine the angular range of the FDFDF quadrupole subsections. Then choose the coil turn number for the transition. Finally adjust the quadrupole coil current to minimize the difference between the SCOFF and the full field model. Because the SCOFF agrees very well with the real fields (see Fig. 12) there is only a small (3\%) increase in the current necessary. Now that the parameters of the realistic gantry model are fixed we are in a position to simulate the performance.

\section{Simulation}

As a reminder our goal here is to understand the extent of the volume (transverse and depth) that can be scanned with minimal beam distortion and while keeping the fields of the AG-CCT fixed.

In our gantry the beam size is defined by the collimator. Therefore we will primarily discuss the simulation results from the collimator to the isocenter. At this point, the beam dynamics between the entrance or coupling point of the gantry and the collimator has not been systematically studied. Due to the large range in momentum, additional steps have to be taken to ensure reasonable transmission through the collimator at all energies. The first and most straightforward option from the beam optics point of view is to place the energy degrader at the location of the collimator. This way the energy of the proton beam passing through this section is fixed and the emittance is only a fraction of the value used in the simulation shown here. Simulation results show that the magnets B1 and B2 in the present form can transport the beam with no significant blurring of the image at the collimator. The feasibility of this option needs to be established by demonstrating that the degrader is compatible with the rotating gantry and that the radiation can be properly shielded. The second option is to add room temperature kickers and quadrupoles that ramp with changing energy to compensate residual dispersion and the change in focusing. The third option is to add sextupole coils in the magnets B1 and B2 to correct the second order dispersion and the chromaticity and, if needed, octupole coils to correct the third order geometrical aberrations generated by the sextupoles. Although no show stopper is expected, detailed study is necessary to confirm that either or some form of combination of these two options actually work.

Let us examine the process of simulating the beam from the collimator to the isocenter. Figure 13 shows the initial phase space at the collimator. In particular there are two plots showing a phase space projection in the in-plane and out-of-plane directions. As the figure shows, the beam is round and the projections are the same in both planes. The emittance of $2 \sigma=30 \pi \mathrm{mm}$-mrad and the orientation is $3 \mathrm{~mm} \times 10 \mathrm{mrad}$. The next step is looking at how the phase space evolves at the isocenter in the case that the sweeper magnets are powered off.

\section{The effect and compensation of spherical aberrations}

The first tracking result that we present is a case where in addition to the sweeper magnets being off, the beam is assumed to have no momentum deviation, (i.e., $\Delta p / p=0$ ). Figure 14 shows the phase space at the isocenter for in plane (left) and out of plane (right). There are a number of things to point out.

Let us begin by looking at the in plane [Fig. 14 (left)]. The first is that there are two sets of points per figure-one with octupole correction and the other without. The similarity shows the octupole correction has little importance in this plane. 

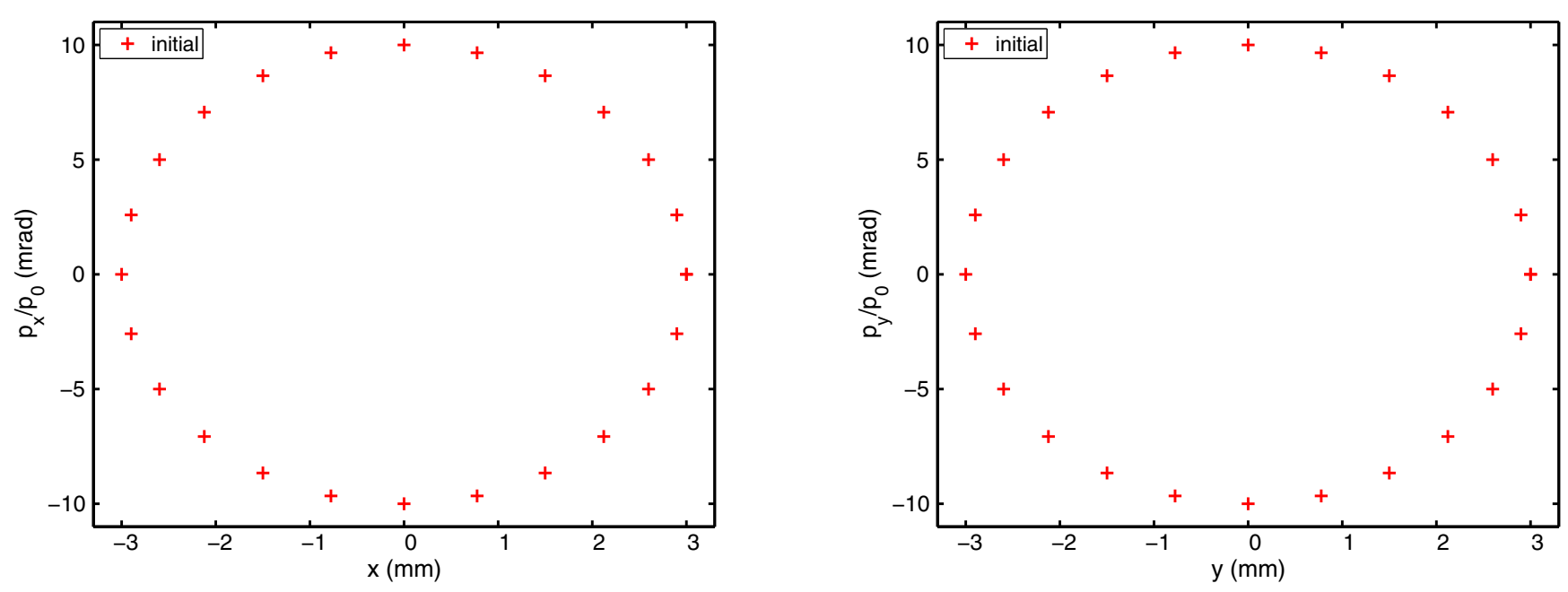

FIG. 13. Initial in-plane (left) and out-of-plane (right) phase space at the collimator. $30 \pi \mathrm{mm}$-mrad.

The extent of the phase space is a little less than $\pm 6 \mathrm{~mm}$ and a little more than $\pm 6 \mathrm{mrad}$ - showing the $1.9 \mathrm{mag}$ nification from the collimator to the isocenter. One also sees the phase space is slightly tilted. This results from the fact that even though the isocenter is at an imaging point of the collimator, it is not at a waist (i.e., $R_{21} \neq 0$ and $\mathrm{R}_{43} \neq 0$ ).

Now let us look at the out of plane [Fig. 14 (right)]. The picture here is quite different. Note the big difference between phase space with and without octupole corrections. Without corrections the phase space is very distorted. However it is nearly perfectly recovered and identical with the in-plane phase space with octupole corrections.

The main cause of the initial distortion is a kinematic effect and due to a combination of two things-the first being that we have a very large emittance and the second that the beam size goes through QO3 with a large offset

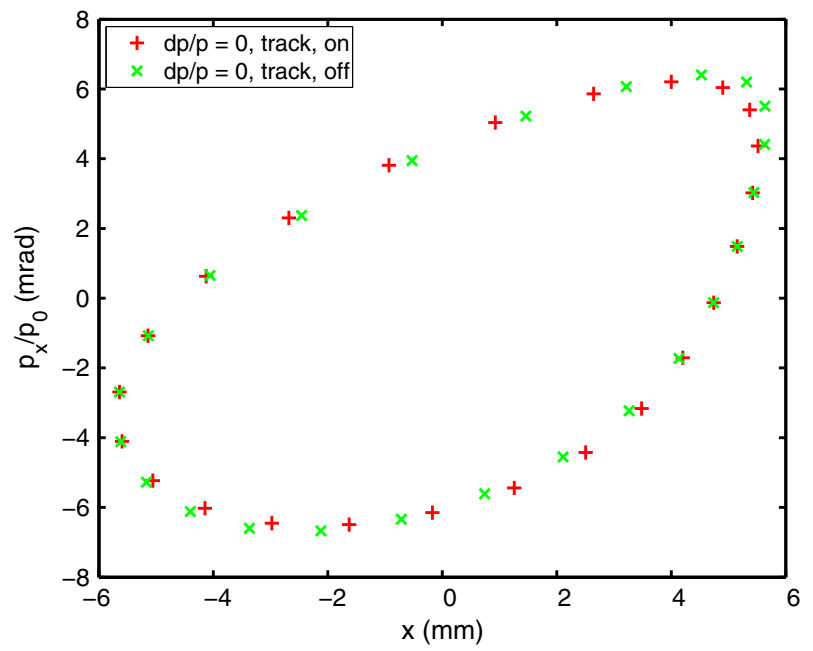

and divergence (see Fig. 5). Any additional phase space distortion coming from B3 is very small. This is one of the main challenges of requiring a large emittance. The good news is that this distortion is low order and can be corrected very effectively with octupoles.

Next let us look at what happens for large momentum deviations. In our case the momentum is assumed to be $20 \%$ higher. Before tracking the quadrupoles and octupoles are adjusted with the fields of the B3 AG-CCT magnets kept the same. The quadrupoles are set to have the same imaging conditions as the case on-momentum case. Also the octupoles are readjusted to compensate low order spherical aberrations. In addition the in-plane sweeper magnet is adjusted to compensate for centroid shift due to second order dispersion.

The results of phase space tracking can be seen in Fig. 15. On top is the on-momentum $(\Delta p / p=0 \%)$

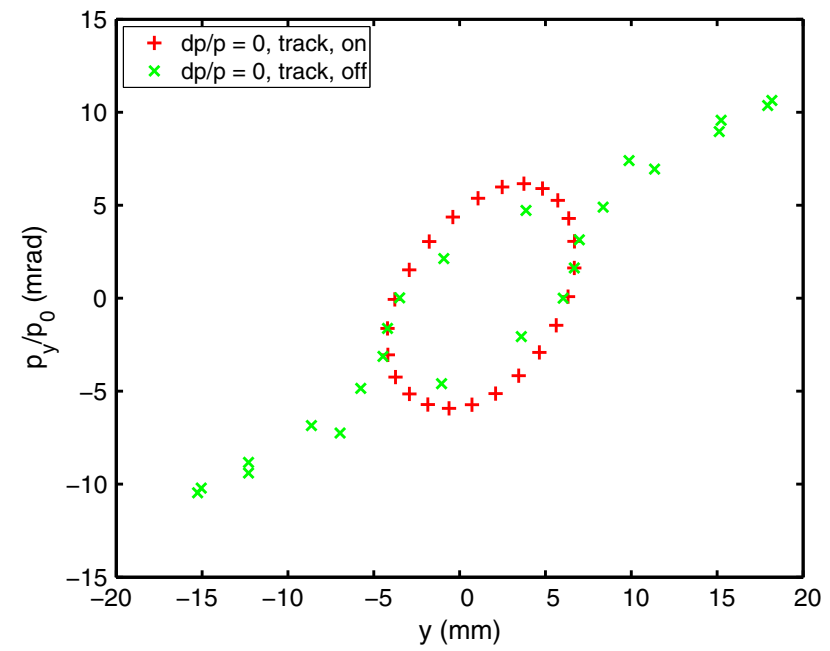

FIG. 14. Final in-plane (left) and out-of-plane (right) phase space at the isocenter. The importance of the three octupole corrections in the out-of-plane phase space is very dramatic. Note the scale of the plots is not the same. 

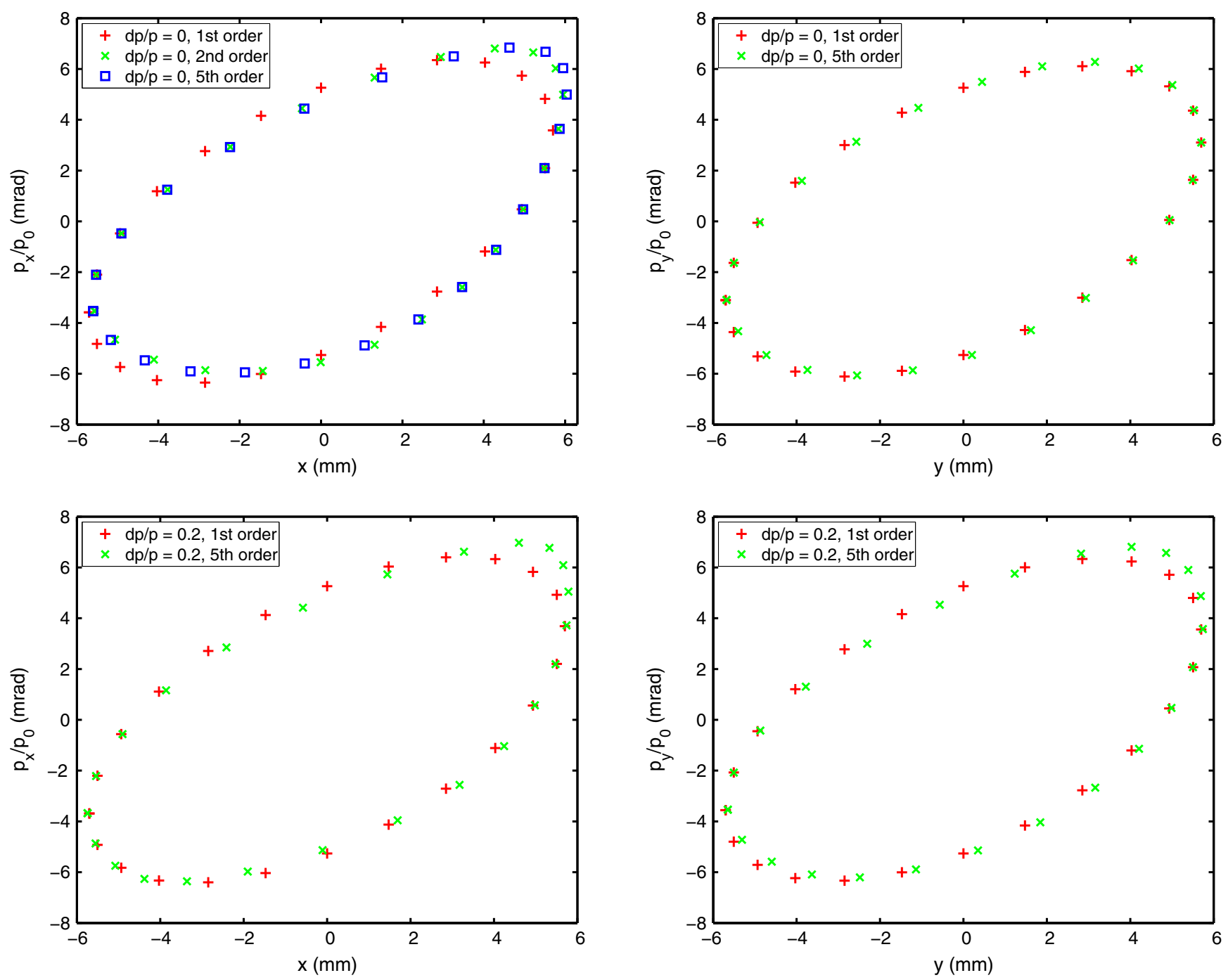

FIG. 15. Final in-plane and out-of-plane phase space at the collimator for $\Delta p / p=0 \%$ and $20 \%$.

in-plane (left) and out-of-plane (right) phase spaces at the isocenter. These are just the same as shown in Fig. 14 with octupole corrections. The bottom two plots in Fig. 15 show the off-momentum $(\Delta p / p=+20 \%)$ in-plane (left) and out-of-plane (right) phase spaces at the isocenter.

Several important observations can be extracted from looking at Fig. 15. The first is that it is remarkable how similar the on-momentum and off-momentum phase spaces look-especially considering that the momentum difference is very large (20\%). It shows that with octupoles, third order aberrations between the collimator and the isocenter become negligible for all momentum.

The second observation is that whatever aberrations exist are mostly at low order. This can be seen because in these plots [compared to showing the beam transport using brute force tracking (Fig. 14)] results of the tracking are plotted using DA transfer maps calculated to first order (linear) and fifth order. One can see that there is a small difference between the tracking using fifth order and a linear map. The upper left shows that remaining aberrations are of the second order.

The next step is to look at what happens to the beam as it is scanned away from the axis. The first step is to look at a zero emittance beam (i.e., just tracking a particle at the center) to understand the transverse scanning range. After that a full beam is tracked and scanned to understand the beam distortions as a function of both momentum and transverse scanning position.

\section{Tracking results while scanning a zero emittance beam}

In this section we start with a zero emittance beam meaning that at the sweeper magnets there is no position or angular offset. The first step is scanning the beam in the in-plane and out-of-plane directions at several discrete beam momenta $(\Delta p / p=-5 \%, 0 \%, 5 \%, 10 \%, 15 \%, 20 \%)$. The results can be seen in Figs. 16 (in plane) and 17 (out of plane). 


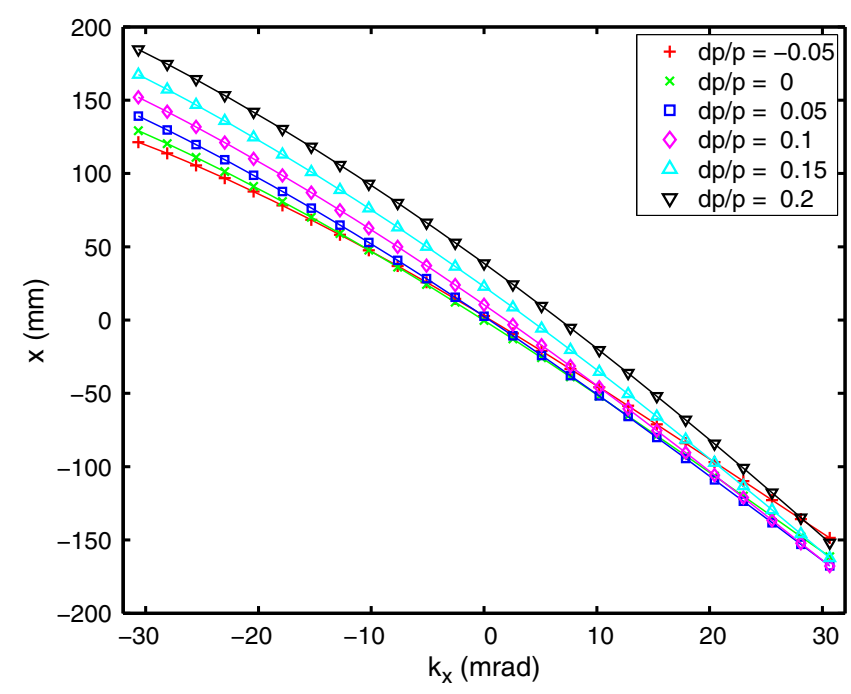

FIG. 16. In-plane offset of the beam center at the isocenter as a function of in-plane sweeper scan angle at different momentum deviations.

The observations are that in the in-plane case there is a large linear field of roughly $\pm 15 \%$. One also sees that there is some second order dispersion (look at the offset of the beam at zero sweeper field setting as a function of momentum). One of the reasons that the response in plane is linear over a large range is that inside the magnet the trajectory does not extend very far from the center (see Fig. 6).

The out-of-plane case is somewhat different. When looking at the out-of-plane behavior the first observation is that as expected, there is no dispersion. The second observation is that the response of the offset versus sweeper position is more nonlinear in the out-of-plane direction. At smaller sweeper angle settings, the out-of-plane response is

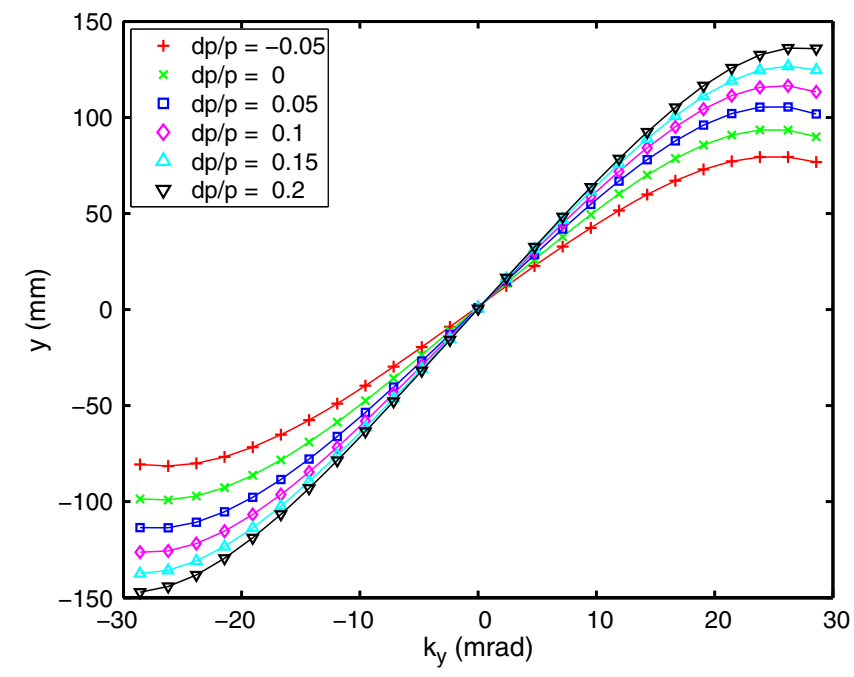

FIG. 17. Out-of-plane offset of the beam center at the isocenter as a function of out-of-plane sweeper scan angle at different momentum deviations. linear. However at the larger sweeper angles, increasing the sweeper field does not increase the amplitude but rather the amplitude turns over and starts to decrease. The largest amplitude reaches $\pm 8 \mathrm{~cm}$ at $\Delta p / p=-5 \%$ to $\pm 14 \mathrm{~cm}$ at $\Delta p / p=+20 \%$. This is due to the large extent of the trajectory in the second to last section of $\mathrm{B} 3$ (second $\mathrm{D}$ in the FDFDF) section (see Fig. 7). This aberration is mostly due to kinematic effects as well as the nonlinearity of the B3 fields. The location of the turnover point continues to drop even as one goes to even lower momenta. In other words the out-of-plane fields become smaller as relative momentum is lowered. As a result for the study we chose our scanning range from $\Delta p / p=-5 \%$ to $\Delta p / p=+20 \%$ where the fields should be relatively large.

Scanning asymmetrically in momentum (from $\Delta p / p=$ $-5 \%$ to $\Delta p / p=+20 \%$ ) relative to the setting of $\mathrm{B} 3$ is advantageous. It means that to scan up to $220 \mathrm{MeV}$ does not mean that the fields need to be set for that energy but at a value that is $20 \%$ lower in field. This reduces the maximum fields of the magnets, and thus the difficulty of the superconducting magnet design.

With a total range of $\Delta p / p=25 \%$ it is possible to cover the full depth range of 4 to $30 \mathrm{~cm}$ with just three AG-CCT magnet settings. This is shown in Fig. 18. Next we present zero emittance scanning as a function of energy. The scans are done with a grid spacing (even in sweeper angles) of $(\Delta p / p=-5 \%, 0 \%, 5 \%, 10 \%, 15 \%, 20 \%)$. The results for two cases $(\Delta p / p=0 \%, 20 \%)$ can be seen in Fig. 19. The axis scaling of the two cases is different. In each figure, a box is drawn corresponding to a scanning area of $23 \mathrm{~cm} \times 14 \mathrm{~cm}$. This scanning range just fits at $\Delta p / p=0$ however fits well inside when $\Delta p / p=+20 \%$.

Also for each plot there is a second set of points corresponding to tracking using a fifth order map. Again one sees good agreement between the map and the brute force tracking giving us confidence that a fifth order map can be used for tracking. For the following plots, the map was used for primary tracking, with cross-checks made with brute force tracking. The reason being that the brute force tracking is significantly slower.

Now that we have an indication of the field sizes that are possible, the next step is to look at distortion of the beam.

\section{Tracking results while scanning a distribution (nonzero emittance)}

In this section we simulate scanning a beam in $3 \mathrm{D}$ to study beam distortion. For the initial configuration, 192 points are launched from the collimator assuming a round beam with a $3 \mathrm{~mm} \times 10 \mathrm{mrad}$ phase space oriented with 16 angles with respect to the $x-y$ plane. Tracking was done with monochromatic beams at six different momentum values $(\Delta p / p=-5 \%, 0 \%, 5 \%, 10 \%, 15 \%, 20 \%)$.

There are choices in how extensive one varies the normal conducting sweeper magnets and the matching quadrupoles and octupoles while scanning. Because the sweeper 


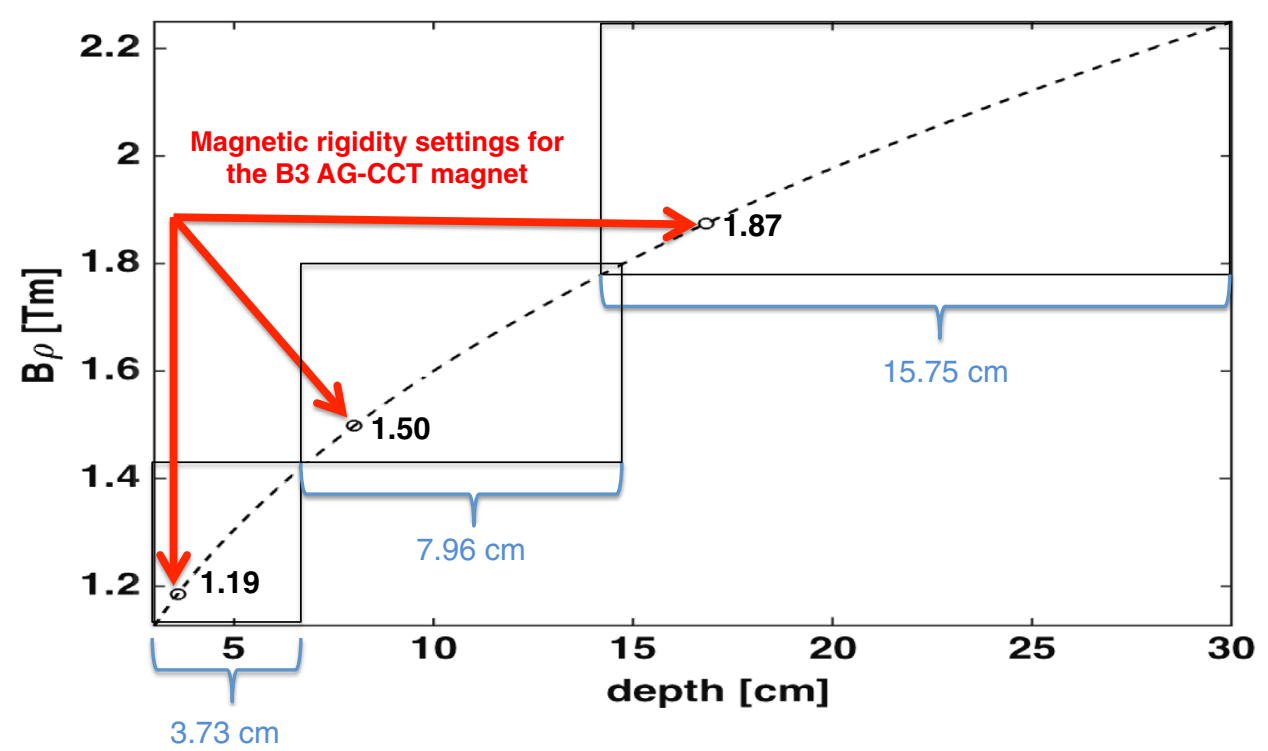

FIG. 18. This is an illustration that with a $25 \%$ momentum acceptance three settings allow one to cover the range of 4 to $30 \mathrm{~cm}$.

magnets are varied at each point, it is very straightforward to add a correction term correcting for uneven grid spacing as well as compensate for second order dispersion. For the quadrupoles and octupoles in the matching section we define three cases. We refer to them as no correction, minimal correction, and optimal correction.

No correction.-Quadrupoles and octupoles do not change during either momentum or sweeper scanning.

Minimal correction.-Quadrupoles and octupoles are fixed at each value of momentum.

Optimal correction.-Quadruples and octupoles are adjusted for each scanning point to correct the imaging and lower order spherical aberration terms.

Next, the tracking results showing the beam distortion are presented initially for minimal correction and then for optimal correction.

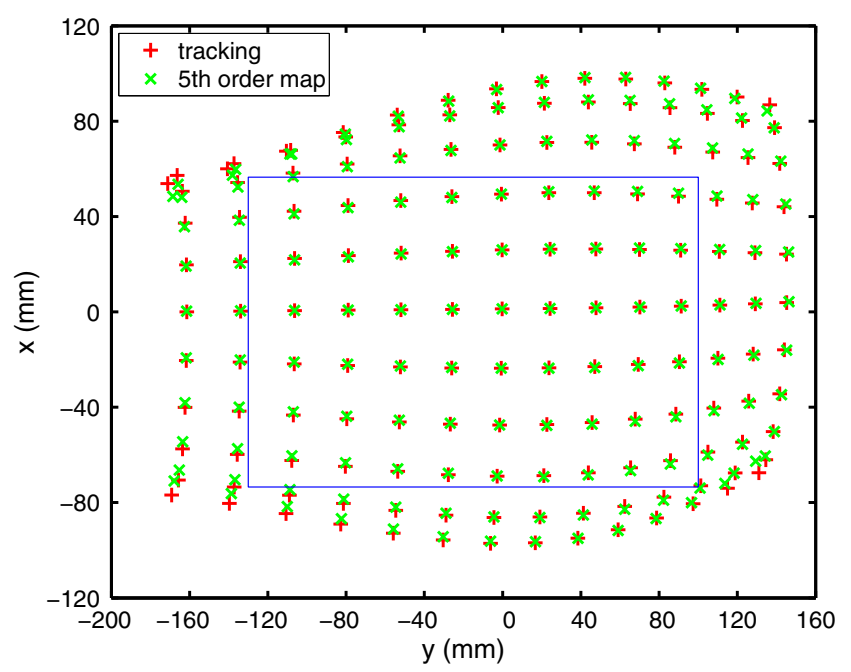

In the case of minimal correction the results can be seen in Fig. 20. There are six plots corresponding to the six values of momenta. For each value of momenta the quadrupoles and octupoles stay fixed and only sweeper magnets change. The size of the field is $23 \mathrm{~cm} \times 10 \mathrm{~cm}$ in the case of $\Delta p / p=-5 \%$, and $23 \mathrm{~cm} \times 14 \mathrm{~cm}$ in the case of $\Delta p / p=0 \%, 5 \%, 10 \%, 15 \%, 20 \%$. There are several observations here. The size of the scanning field is large horizontally and somewhat smaller vertically. As compared to the requirements listed in Table I, the vertical size is a bit smaller than desirable (but still larger than the size of PSI Gantry 2 for example).

There is a desire to minimize beam distortion for better dose distributions and simpler treatment planning. One also sees the beam "water marks" indicating the changes in size and some distortion of the beam. Clearly there is distortion

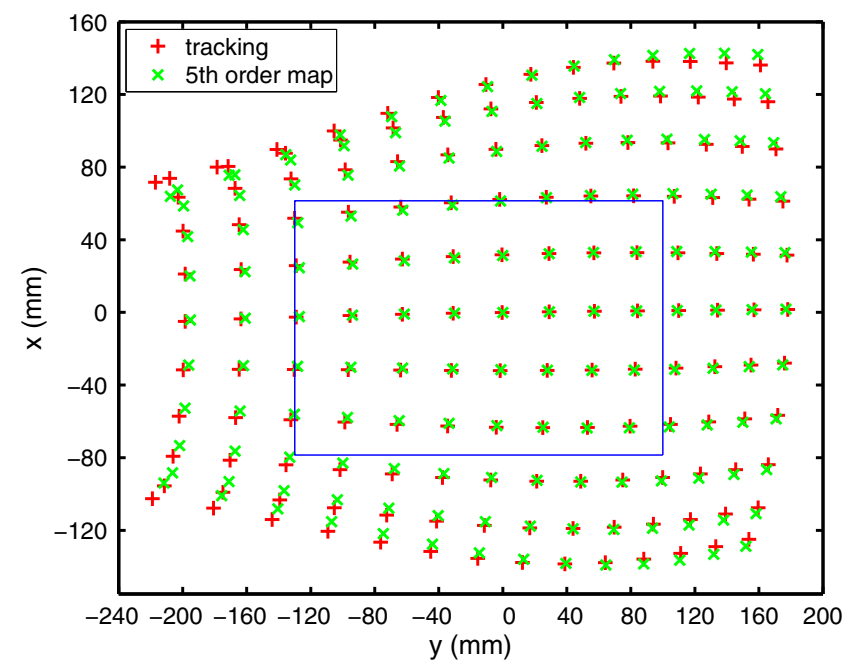

FIG. 19. Tracking is done at $\Delta p / p=0$ (left) and at $\Delta p / p=20 \%$ (right). The plots show that brute force tracking and tracking using fifth order map agree very well. 

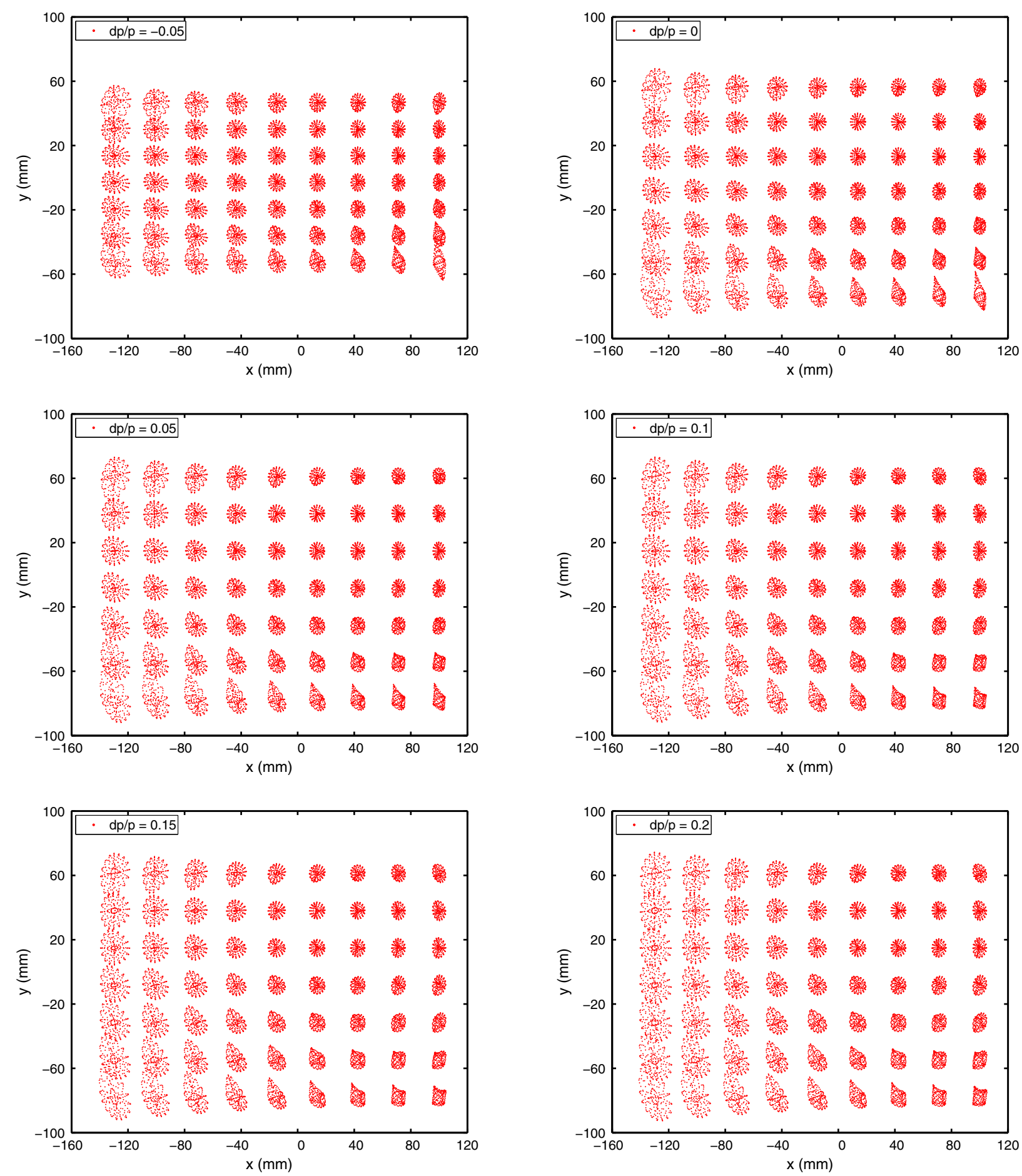

FIG. 20. Grid scans with minimal correction for $\Delta p / p=-5 \%, 0 \%,+5 \%,+10 \%,+15 \%,+20 \%$. Note the rectangular grid size of $23 \mathrm{~cm} \times 10 \mathrm{~cm}$ for $\Delta p / p=-5 \%$ and $23 \mathrm{~cm} \times 14 \mathrm{~cm}$ for $\Delta p / p \geq 0 \%$.

over the field. In this paper we have not quantified nor analyzed how this might impact the treatment. Nevertheless, there are ways to minimize the distortion by using the optimal correction which we now present.

The results for optimal correction can be seen in Fig. 21 . There are six plots that correspond to the six values of momenta. In the optimal case the quadrupoles and octupoles are adjusted for each point. There are several observations here. The size of the scanning region remains mostly the same as is expected (there is a slight degradation in the upper and lower left points for $\Delta p / p=0 \%)$. However there is nearly no distortion over the scanning field.

This is a demonstration that one can scan over a relatively large transverse field on the order of 

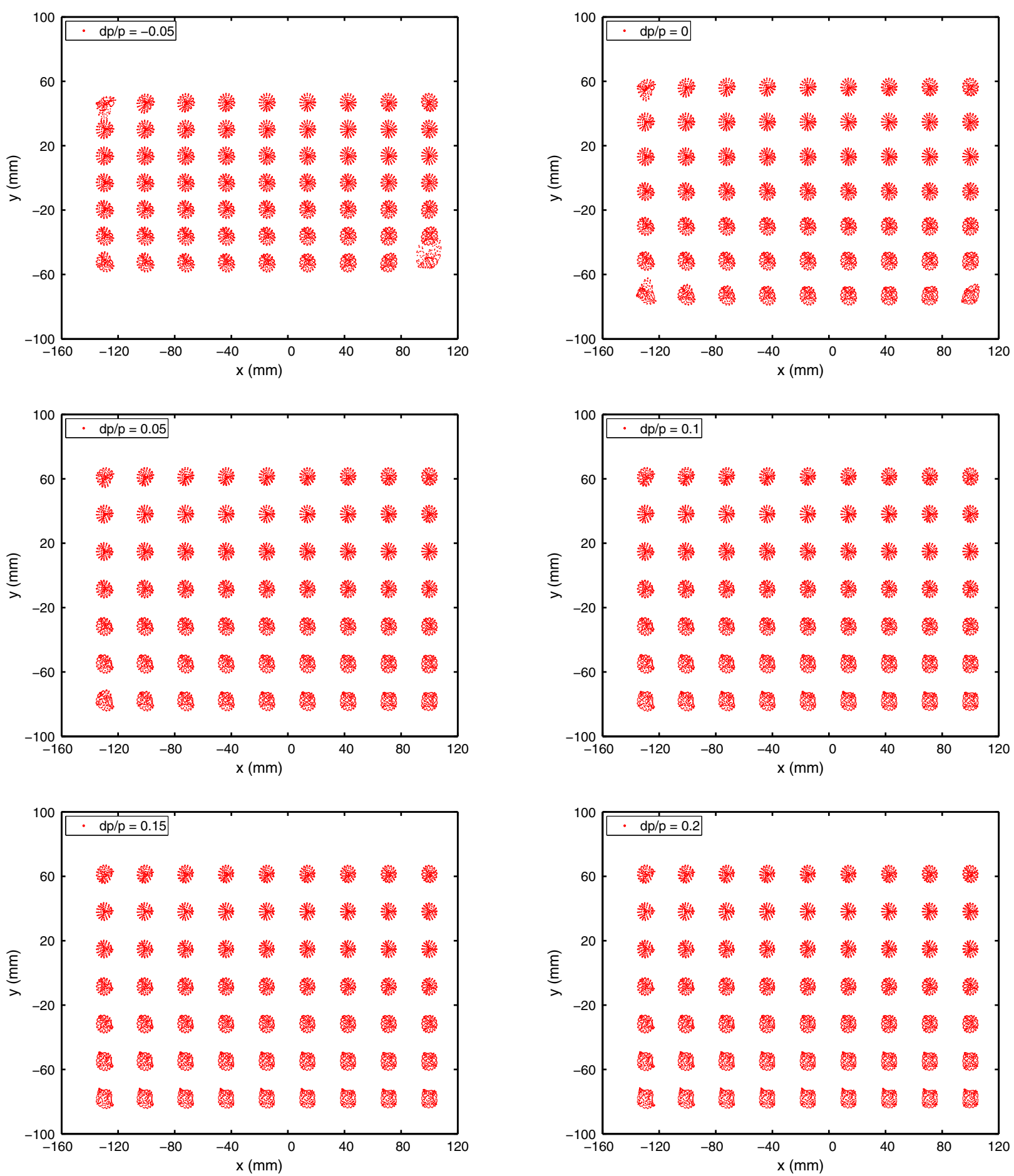

FIG. 21. Grid scans with full correction for $\Delta p / p=-5 \%, 0 \%,+5 \%,+10 \%,+15 \%,+20 \%$. Note the rectangular grid size of $23 \mathrm{~cm} \times 10 \mathrm{~cm}$ for $\Delta p / p=-5 \%$ and $23 \mathrm{~cm} \times 14 \mathrm{~cm}$ for $\Delta p / p \geq 0 \%$.

$23 \mathrm{~cm} \times 14 \mathrm{~cm}$ and over a $25 \%$ momentum range with minimal beam distortion and without changing the fields of the superconducting AG-CCT magnets. This is a significant achievement, showing the feasibility of a gantry design using locally achromatic AG-CCT superconducting magnetic section. All goals set out in Table I were mostly met or exceeded.

\section{Out-of-plane assist: The potential for even larger scanning fields}

The question is can one do even more. A potential area of improvement would be to increase the usable out-of-plane scanning region. As described in Table I it would be desirable to have a scanning region in which the scanning field is greater than $20 \mathrm{~cm} \times 20 \mathrm{~cm}$. Towards this end an 


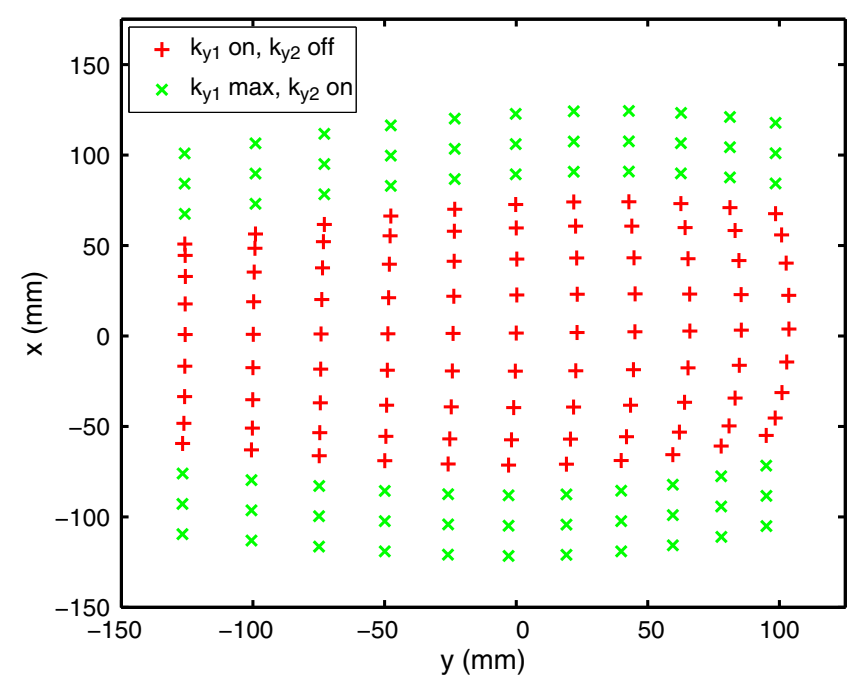

FIG. 22. Extension of the out-of-plane scanning range at $\Delta p / p=-5 \%$ using a second out-of-plane sweeper magnet located just down stream of B3.

addition to the gantry design is made. A second out-ofplane sweeper magnet (called an out-of-plane assist sweeper) is included downstream of B3 to extend the out-of-plane scanning field size. The results for the zero emittance case can be seen in Fig. 22. The condition plotted is one where the momentum is set to $\Delta p / p=-5 \%$ (the momentum that had corresponded with the smallest field size). In the plot the red dots show the extent of the scanning using just the original upstream out-of-plane sweeper. The green dots show how the field can be extended by keeping the out-of-plane sweeper at its maximum value and powering the downstream out-of-plane sweeper up to about $\pm 50 \mathrm{mrad}$. The out-of-plane scanning field is extended by nearly a factor of 2 .

TABLE X. Summary of simulation results for a gantry without and with an out-of-plane assist (OOPA) sweeper.

\begin{tabular}{lcc}
\hline \hline Parameter & No OOPA & With OOPA \\
\hline Proton acceptance $(2 \sigma)(\pi$ mm-mrad) & 30 & 30 \\
Beam size at isocenter $(2 \sigma)(\mathrm{mm})$ & 5.7 & 5.7 \\
Beam energy range $(\mathrm{MeV})$ & 70 to 220 & 70 to 220 \\
Space from the last magnet & 1.25 & $(1.25-1.5)^{\mathrm{a}}$ \\
$\quad$ to the isocenter $(\mathrm{m})$ & & \\
In-plane SAD $(\mathrm{m})$ & 3.89 & 3.89 \\
Out-of-plane SAD (m) & 3.13 & $<3.13^{\mathrm{b}}$ \\
Transverse scanning field (cm $\left.{ }^{2}\right)$ & $20 \times 14$ & $>(25 \times 20)$ \\
Momentum range w/o ramping & & \\
Maximum sweeper angle (mrad) & \pm 30 & \pm 50 \\
Momentum range w/o ramping & & \\
superconducting magnets $(\%)$ & $\pm 25 \%$ & $> \pm 25 \%$ \\
\hline \hline
\end{tabular}

\footnotetext{
${ }^{\mathrm{a}}$ Depending if the gantry radius needs to be increased to make room for the downstream sweeper magnet.

${ }^{\mathrm{b}}$ Depends how the different sweeper magnets are excited.
}

Of course to realize such a scheme would require an understanding of the realistic range of a large aperture downstream sweeper magnet. Other considerations are whether there is enough room to include such a magnet without increasing the radius of the gantry. The vertical SAD does decrease but still remains above $2 \mathrm{~m}$. Of course this is less impactful on the skin dose than if both the inplane and out-of-plane SADs decreased [29]. The advantages of doubling the size of the scanning region are very attractive. Finally Table X shows the results of the study. The results shown are with and without the second out-ofplane assist sweeper. They can be compared with the desired functional requirements in Table I.

\section{SUMMARY REMARKS}

In summary we have presented a new magnet design concept, the LR-CCT and its quadrupole version AG-CCT, which is compatible with an achromatic layout. We have demonstrated their advantages in the application in a proton therapy gantry. We have optimized such a gantry design for application of 3D pencil beam scanning and have used locally achromatic superconducting sections that provide a large momentum acceptance $(\Delta p / p \approx 25 \%)$. The optical and dynamic performance have been analyzed using the $3 \mathrm{D}$ field derived from the actual windings of the AG-CCT simulated with the full equations of motion. The results show that with appropriate higher order correction, a 3D volume (large transverse scanning field and up to $\Delta p / p=25 \%)$ can be scanned with little beam shape distortion-all while keeping the AG-CCT field fixed. This demonstrates that it is possible to obtain fast 3D scanning, while minimizing the need for fast field ramping of the superconducting magnets-addressing a major technical risk associated with implementing superconducting technology in medical gantries. For proton gantries the corresponding superconducting magnet system holds promise of dramatic reduction in weight. For heavier ion gantries there may furthermore be a significant reduction in size.

It is worth noting that we do not quantify the issues of magnet imperfections, tolerances, and correction schemes. Possible source of errors are: geometric, manufacturing, magnetization, hysteresis, snap-back, etc. These errors can give rise to magnetic field distortions resulting in errors in orbit dispersion, profile, etc. These effects and their mitigation will be the subject of a future study.

In this paper we discussed the application of the AGCCT. However, in its most general form, the LR-CCT concept provides a method of rapidly switching the sign of any multipole-dipole, quadrupole, sextupole, etc. As a result, the LR-CCT magnet concept can be applied to a wide variety of systems requiring (i) the transport of charged particle beams over a large momentum range (such as gantries or accelerators) and (ii) the transport of 
beams with a large energy spread, as may emanate, for example, from Advanced Accelerator sources.

It is conceivable that the LR-CCT concept can be applied to design the magnets of the conventional FFAG type of gantries. Furthermore, the LR-CCT can be applied to a broad spectrum of applications in accelerator and x-ray radiation source design.

\section{ACKNOWLEDGMENTS}

Some of the authors (W. W., L. B., S. C., S. P. and D. R.) would like to express our deep thanks to Andy Sessler who we had the fortune of working closely with for many years towards the goal of developing technology for ion beam therapy. This work is supported under U.S. Department of Energy under Contract No. DE-AC02-05CH11231 and National Science Foundation under Grant No. DGE 1106400.

[1] E. Pedroni, E. Bacher, H. Blattmann, T. Boehringer, A. Coray, and A. Lomax, Med. Phys. 22, 37 (1995).

[2] J. M. Schippers, V. Vrankovic, and D. George, Nukleonika 48, S145 (2003).

[3] J. Schippers, V. Vrankovic, and D. George, in Proceedings of the 17th International Conference on Cyclotrons and Their Applications (PASJ, Tokyo, Japan, 2004), p. 262.

[4] E. Pedroni et al., Z. Med. Phys. 14, 25 (2004).

[5] R. Fuchs, U. Weinrich, and P. Emde, in Proceedings of the 9th European Particle Accelerator Conference, Lucerne, 2004 (EPS-AG, Lucerne, 2004), pp. 2550-2552.

[6] U. Weinrich, in Proceedings of the 10th European Particle Accelerator Conference, Edinburgh, Scotland, 2006 (EPS-AG, Edinburgh, Scotland, 2006), pp. 964-966.

[7] A. Gerbershagen, D. Meer, J. M. Schippers, and M. Seidel (unpublished).

[8] D. Robin, D. Arbelaez, S. Caspi, C. Sun, A. Sessler, W. Wan, and M. Yoon, Nucl. Instrum. Methods Phys. Res., Sect. A 659, 484 (2011).

[9] K. Mizushima, in Proceedings of the 2nd International Particle Accelerator Conference, San Sebastián, Spain (EPS-AG, Spain, 2011), pp. 3603-3605.

[10] Y. Iwata et al., in Proceedings of the 2nd International Particle Accelerator Conference, San Sebastián, Spain (Ref. [8]), pp. 3601-3603.
[11] Y. Iwata et al., Phys. Rev. ST Accel. Beams 15, 044701 (2012).

[12] K. Noda et al., Nucl. Instrum. Methods Phys. Res., Sect. B 331, 6 (2014).

[13] J. M. Cameron, V. Anferov, and T. A. Antaya, Compact isocentric gantry, Patent No. US20110101236A1, 2010.

[14] V. Derenchuk, The pronova sc360 gantry, at the Modern Hadron Therapy Gantry Developments, Cockcroft Institute, 2014 (unpublished).

[15] W. Wan, D. Robin, A. Sessler, and C. Sun, in Proceedings of the 3rd International Particle Accelerator Conference, New Orleans, LA, 2012 (IEEE, Piscataway, NJ, 2012), pp. 4100-4102.

[16] D. Trbojevic, B. Parker, E. Keil, and A. M. Sessler, Phys. Rev. ST Accel. Beams 10, 053503 (2007).

[17] D. Trbojevic, B. Baker, and M. Pullia, in Proceedings of the 25th Particle Accelerator Conference, PAC-2013, Pasadena, CA, 2013 (IEEE, New York, 2013), pp. 1119-1121.

[18] S. Caspi, D. Arbelaez, L. Brouwer, D. R. Dietderich, H. Felice, R. Hafalia, S. Prestemon, D. Robin, C. Sun, and W. Wan, Nucl. Instrum. Methods Phys. Res., Sect. A 719, 44 (2013).

[19] D. Meyer and R. Flasck, Nucl. Instrum. Methods Phys. Res., Sect. A 80, 339 (1970).

[20] C. Goodzeit, M. Ball, and R. Meinke, IEEE Trans. Appl. Supercond. 13, 1369 (2003).

[21] C. L. Goodzeit, R. B. Meinke, and M. Ball, Concentric tilted double-helix dipoles and higher-order multipole magnets, Patent No. US6921042B1, 2005.

[22] C. Goodzeit, R. Meinke, and M. Ball, in Proceedings of the 22nd Particle Accelerator Conference, PAC-2007, Albuquerque, NM (IEEE, New York, 2007), pp. 560-562.

[23] R. Meinke, Conductor assembly formed about a curved axis, Patent No. US7889046B2, 2008.

[24] A. V. Gavrilin, M. D. Bird, V. E. Keilin, and A. V. Dudarev, IEEE Trans. Appl. Supercond. 13, 1213 (2003).

[25] S. Caspi et al., IEEE Trans. Appl. Supercond. 24, 4001804 (2014).

[26] L. Brouwer, D. Arbelaez, S. Caspi, H. Felice, S. Prestemon, and E. Rochepault, IEEE Trans. Appl. Supercond. 24, 4001506 (2014).

[27] M. Berz and K. Makino, Michigan State University Technical Report No. MSUHEP, 060804, 2011.

[28] I. Kubler, Master's thesis, Justus Liebig Universität, Giessen, 6300 Giessen, FRG, 1987.

[29] H. Vrenken, R. Schuitema, O. C. Dermois, and J. M. Schippers, Nucl. Instrum. Methods Phys. Res., Sect. A 426, 618 (1999). 